\title{
Biculturalisme et revendications culturelles et identitaires : ce que révèlent les pratiques artistiques contemporaines des femmes māori
}

Catherine Pellini

\section{CpenEdition}

\section{Journals}

Édition électronique

URL : https://journals.openedition.org/tc/7595

DOI : $10.4000 /$ tc. 7595

ISBN : 0248-6016

ISSN : 1952-420X

Éditeur

Éditions de l'EHESS

Édition imprimée

Date de publication : 24 décembre 2015

Pagination : 234

ISBN : 978-2-7132-2505-5

ISSN : 0248-6016

Référence électronique

Catherine Pellini, «Biculturalisme et revendications culturelles et identitaires : ce que révèlent les pratiques artistiques contemporaines des femmes māori », Techniques \& Culture [En ligne], 64 | 2015, mis en ligne le 24 mars 2016, consulté le 29 septembre 2022. URL : http://journals.openedition.org/tc/ 7595 ; DOI : https://doi.org/10.4000/tc.7595

Ce document a été généré automatiquement le 29 septembre 2022.

Tous droits réservés 


\title{
Biculturalisme et revendications culturelles et identitaires : ce que révèlent les pratiques artistiques contemporaines des femmes māori
}

\author{
Catherine Pellini
}

\section{NOTE DE L'ÉDITEUR}

Nous vous invitons à consulter la synthèse de cet article (édition papier) en téléchargeant le document placé en annexe.

Le champ de la création artistique contemporaine connaît un phénomène d'expansion au sein des peuples autochtones du Pacifique depuis plus de deux décennies (Thomas 1999 ; Graille 2003 ; Glowczeswski et Henry 2007). Les productions des artistes māori contemporains de Nouvelle-Zélande s'inscrivent dans ce contexte tout en reflétant les particularités d'une société qui a connu la colonisation britannique au XIXe siècle mais qui se veut biculturelle en s'appuyant pour cela sur un traité établi en 1840 entre les colons et les Māori : le traité de Waitangi. Ce dernier était censé garantir les droits des Māori, en particulier la propriété des terres, mais il fut rapidement bafoué et les Māori virent la plupart de leurs terres être confisquées ou achetées à très bas prix. Après avoir connu de profondes mutations sociétales, la population māori ne représente plus aujourd'hui que $15 \%$ des 4200000 habitants de cette île du Sud-Ouest de l'océan Pacifique (Statistics New Zealand 2013). Bien qu'indépendant depuis 1947, l'État néozélandais est encore fortement marqué par son passé colonial. Les Māori ont subi dans un premier temps une politique d'assimilation mais, depuis les années 1970, ils s'appuient notamment sur le traité de Waitangi, et plus particulièrement sur la version rédigée en māori ${ }^{1}$, pour faire reconnaître leurs droits, leur culture et leur identité (Smith 1999; Walker 2004). Ils ont été nombreux à lutter pour l'instauration d'une société biculturelle telle que définie par Schwimmer (1972 [1968]) : une société au sein 
de laquelle les Māori auraient accès à une " pleine citoyenneté » et participeraient ainsi à la vie sociale et politique néo-zélandaise sans pour autant être « assimilés » et donc devoir renoncer à la culture et à l'identité māori.

1 Les œuvres des artistes contemporains māori matérialisent souvent le biculturalisme car elles mêlent des matériaux, des techniques, des motifs, des styles et des sujets issus des cultures māori et pākehā ${ }^{2}$. Elles sont souvent réalisées par des artistes ayant suivi un apprentissage dans des institutions telles que les Écoles des beaux-arts ou ayant au moins été scolarisés dans des écoles «à l'occidentale ». Ils se distinguent ainsi de ceux pratiquant l'art «traditionnel ${ }^{3}$ par un mode d'apprentissage différent, l'emprunt de techniques, de styles et de matériaux à des tendances artistiques internationales et des sujets marqués par un mode de vie urbain et l'existence dans une société (post)coloniale (Thomas 1995 ; Charles-Rault 2005). Depuis les années 1990, ces artistes ont accès à des espaces d'exposition autrefois réservés aux arts occidentaux, ce qui marque à la fois la reconnaissance de leur talent à l'échelle individuelle mais également la reconnaissance de la culture contemporaine māori (McCarthy 2007) par les Pākehā. Leurs œuvres symbolisent le lien de leurs auteurs avec la tradition artistique māori tout en présentant des aspects novateurs issus de l'influence occidentale (Ihimaera, Adsett et Whiting 1996 ; Thomas 1995 ; Graille 2003).

2 Le changement paraît encore plus important dans le cas des femmes puisque, avant les années 1950, les seuls objets à caractère esthétique qu'elles réalisaient étaient des tissages. Les productions sculptées et peintes étaient faites par les hommes car les femmes possèdent un caractère "noa » (terme parfois traduit par " profane ») qui était pensé comme incompatible avec l'intervention des esprits nécessaire à la bonne réalisation des œuvres (Best 1924: 255 ; Thomas ibid: 67). Suite aux bouleversements engendrés par la colonisation et notamment au phénomène d'urbanisation, les femmes māori ont diversifié leurs pratiques artistiques. Elles ont commencé à être reconnues sur la scène artistique néo-zélandaise en même temps que les artistes māori masculins à partir des années 1950, notamment grâce à l'initiative de Gordon Tovey, le responsable du programme d'enseignement artistique au sein du ministère de l'Éducation de l'époque. Gordon Tovey avait décidé de recruter des artistes māori pour promouvoir l'apprentissage de l'art māori dans les écoles (Smith 2001 : 93). Parmi ces artistes, plusieurs femmes ont acquis une notoriété dans le champ de l'art contemporain néo-zélandais, parfois même international, et ont ouvert la voie à de nouvelles générations d'artistes. C'est à ces dernières que je me suis intéressée durant une enquête de terrain doctorale d'un an menée en Nouvelle-Zélande (de décembre 2012 à novembre 2013). J'ai rencontré plus d'une trentaine de femmes artistes māori âgées de 27 à 65 ans, des artistes aux origines māori et pākehā vivant dans un milieu urbain et se qualifiant elles-mêmes de " contemporary māori artists " et/ou exposant dans des espaces dédiés à l'art contemporain. Leurs œuvres présentent des caractéristiques biculturelles mais elles sont désignées par leurs auteures comme étant de l'art contemporain māori et non seulement de l'art contemporain ${ }^{4}$. Leurs productions offrent un reflet particulier du contexte socio-culturel dans lequel elles vivent mais leur pratique artistique est aussi envisagée comme un mode d'action véhiculant l'intentionnalité des artistes qui rencontre celle des spectateurs dans des lieux d'exposition particuliers. La notion d'agentivité développée par Alfred Gell (2009) ${ }^{5}$ est donc importante dans ce contexte mais c'est également le cas des études centrées sur les relations existant entre l'art et les mouvements contestataires (Negash 
2004 ; Balasinski et Mathieu 2006 ; Bradley et Esche 2007). En effet, outre l'expression de revendications politiques et culturelles associées à une volonté de décolonisation que l'on trouve également dans de nombreuses œuvres d'artistes māori masculins, les productions féminines répondent fréquemment au désir des artistes de faire connaître les expériences qui leur sont propres en tant que femmes issues d'un peuple colonisé (Diamond 1999, Gwynne 2009). En plus des discriminations liées à leur sexe, les femmes māori peuvent subir des discriminations dues à leur origine et elles n'accordent alors pas toujours la priorité à la lutte contre le sexisme. Elles se trouvent dans une position parfois inconfortable car il leur est difficile de critiquer certains aspects qu'elles ressentent comme allant à l'encontre des femmes à l'intérieur de la société māori. Elles pourraient ainsi être considérées comme déloyales ou renégates et être accusées d'affaiblir les revendications culturelles auxquelles elles adhèrent par ailleurs (Curchin 2011, Simmonds 2011). Ce phénomène est visible dans d'autres sociétés et il est appréhendé par la théorie de l'intersectionnalité. Cette théorie est née du constat qu'il existe différents axes de différenciation sociale - le sexe ou le genre, la classe, la "race ", l'ethnicité, l'âge, les handicaps et l'orientation sexuelle - et que plusieurs régimes d'inégalités peuvent s'entrecroiser et co-évoluer. Ces diverses catégories sont à l'origine de multiples systèmes d'oppression qui produisent et reproduisent des inégalités sociales (Bilge 2009 : 70). Cette idée a été évoquée dès la fin du XIX ${ }^{\mathrm{e}}$ siècle par des féministes noires mais elle n'a vraiment été théorisée qu'à partir des années 1990. Elle connaît aujourd'hui un certain succès car elle peut être appliquée à de nombreux contextes et n'est pas seulement limitée à l'étude des discriminations : elle permet aussi d'examiner les intersections de divers vecteurs de pouvoir ${ }^{6}$. La théorie de l'intersectionnalité invite à considérer les identités multiples des femmes. Elle est donc applicable dans le cas des femmes artistes māori lesquelles ont des origines diverses mais se disent māori, s'inscrivent dans le champ artistique où il n'est pas toujours facile d'être reconnue lorsque l'on est une femme, enfin sont des Māori vivant dans un contexte urbain pākehā. Leurs créations présentent alors un "bricolage " à double niveau. Comme pour les artistes masculins contemporains, leur art puise dans la tradition tout en innovant. Il présente un mécanisme d'emprunt à diverses sources. Des éléments provenant de mondes sociaux différents sont utilisés et reconfigurés dans des créations qui sont très distinctes en fonction de leurs auteures mais qui partagent néanmoins un certain nombre de caractéristiques. Ce premier «bricolage» s'accompagne d'un deuxième cette fois au niveau des identités. Les œuvres et les discours portant sur les parcours de vie des artistes permettent d'appréhender les identités multiples de ces femmes qu'elles concilient en mettant en place diverses stratégies. Afin d'examiner ce double "bricolage", les œuvres et les parcours de vie détaillés de quatre artistes rencontrées sur le terrain vont être présentés. Ils permettront d'aborder des traits particuliers également visibles chez d'autres artistes.

\section{Présentation générale}

Malgré la multiplicité des styles individuels très différents des artistes māori contemporaines, certaines pratiques récurrentes sont privilégiées. Les œuvres peuvent alors être regroupées en sept grandes catégories: impressions sur papier, tissu et autres matériaux ; peintures figuratives et abstraites (surtout à l'huile et à l'acrylique) sur toile et autres supports comme des panneaux de bois; modelages et sculptures en argile; vidéos; photographies; installations multimédias; et enfin tissages réalisés 
avec des matériaux "traditionnels» (fibres végétales) ou contemporains (fibres synthétiques, plastique, aluminium).

3 La majorité des femmes à l'origine de ces œuvres ont étudié dans des établissements scolaires secondaires et universitaires «à l'occidentale ». Elles ont également pour la plupart d'entre elles voyagé aux États-Unis et/ou en Europe et elles s'intéressent à la scène artistique mondiale. Même si elles ne mentionnent pas forcément explicitement l'influence d'artistes autres que néo-zélandais et surtout māori, certaines de leurs œuvres évoquent des mouvements comme l'expressionnisme, le pointillisme ou encore l'art naï. Elles exposent dans des espaces consacrés à l'art contemporain et non exclusivement à l'art māori. Le choix des espaces d'exposition est important car il permet aux artistes de toucher une audience plus ou moins large et variée. Les artistes māori contemporaines essaient de faire connaître leurs œuvres auprès de publics divers, Māori et Pākehā. Certaines d'entre elles s'impliquent beaucoup dans l'organisation des expositions; elles réfléchissent à des scénographies originales afin que leurs œuvres aient un impact maximal sur les spectateurs. Elles manifestent également la volonté de transmettre les savoirs liés à leurs pratiques en animant des ateliers à destination d'enfants et d'adultes, en créant face au public tout en interagissant avec lui.

4 Leurs productions semblent très différentes de ce qui se faisait avant la colonisation. Les Māori réalisaient des créations sculptées de grande envergure: des waka, des pirogues pouvant mesurer plus de trente mètres de long, et des wharenui, des maisons de réunion symbolisant le corps d'un ancêtre (fig. 1), (fig. 2), (fig. 3). Ils sculptaient également de petits objets dans l'os, le bois et la pierre et ils pratiquaient l'art pariétal, les tatouages et les tissages (Best 1924; Barrow 1968; Thomas 1995). Ces derniers étaient surtout exécutés par des femmes: il s'agissait des principales réalisations féminines précoloniales à caractère esthétique. La distinction genrée des tâches est généralement expliquée par le fait que toutes les réalisations importantes faisaient intervenir des atua, - des esprits - ; les grandes productions sculptées ne faisant pas exception à la règle. L'objectif d'efficacité auquel ces dernières devaient répondre ne pouvait être garanti qu'en s'assurant de l'intervention bienveillante d'entités surnaturelles au long du processus de fabrication. Les lieux, les objets et les personnes impliqués dans ces activités devenaient tapu (sacrés) et étaient entourés de nombreuses restrictions (Best 1924 : 255-256). Les femmes possédant un caractère noa ${ }^{7}$, - propre à désacraliser les choses -, il leur était interdit d'approcher des lieux de création des réalisations sculptées et plus encore de les produire (Best 1924:255; Thomas 1995:67; King $2003:$ : 86). En revanche, l'origine et la transmission du tissage aux humains, liées à des divinités féminines pouvant diverger suivant les récits - Hine-rauamoa (Tamarapa et Wallace 2013: 3), Hine-teiwaiwa (Hamilton-Pearce 2009: 39-40) et Niwareka (Ihimaera, Adsett et Whiting 1996: 13) - font du tissage une pratique traditionnellement associée aux femmes. Si, comme nous le verrons par la suite, le tissage est encore une activité très importante pour certaines artistes contemporaines, nombre d'entre elles font référence également aux réalisations peintes et sculptées habituellement par les hommes, tout en mêlant ces éléments māori à d'autres, issus cette fois de la culture artistique occidentale. 
Waka

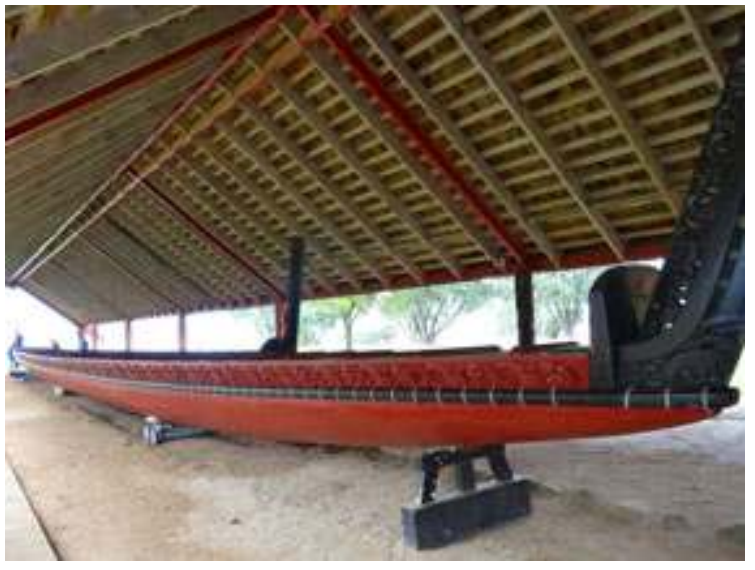

Waitangi

(C) C. Pellini, 5 juin 2013

Extérieur d'une whare whakairo

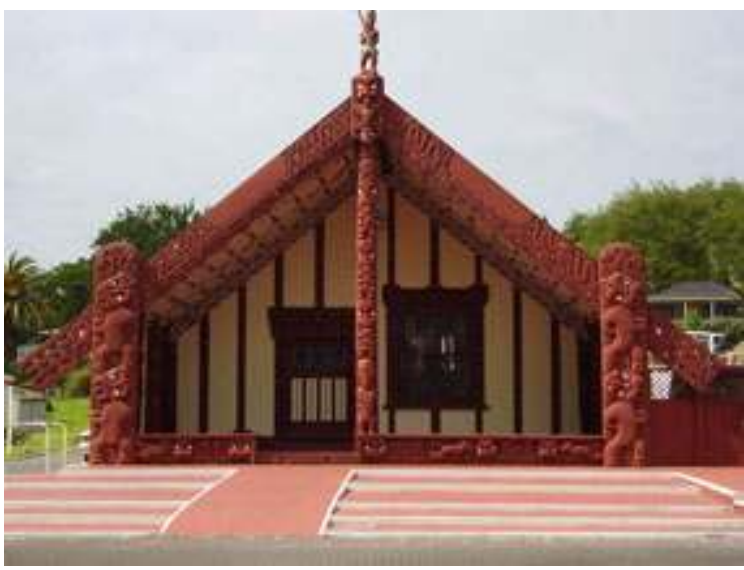

Rotorua

(c) C. Pellini, 3 janvier 2013

Intérieur d'une whare whakairo

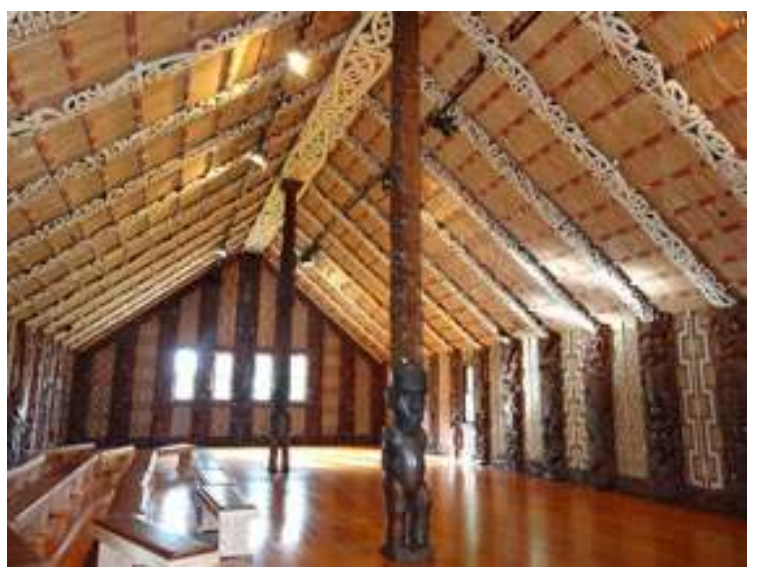

Waitangi

(c) C. Pellini, 5 juin 2013 


\section{La peinture au service de revendications politiques et culturelles : l'exemple de Tracey Tawhiao}

\section{In Love Air}

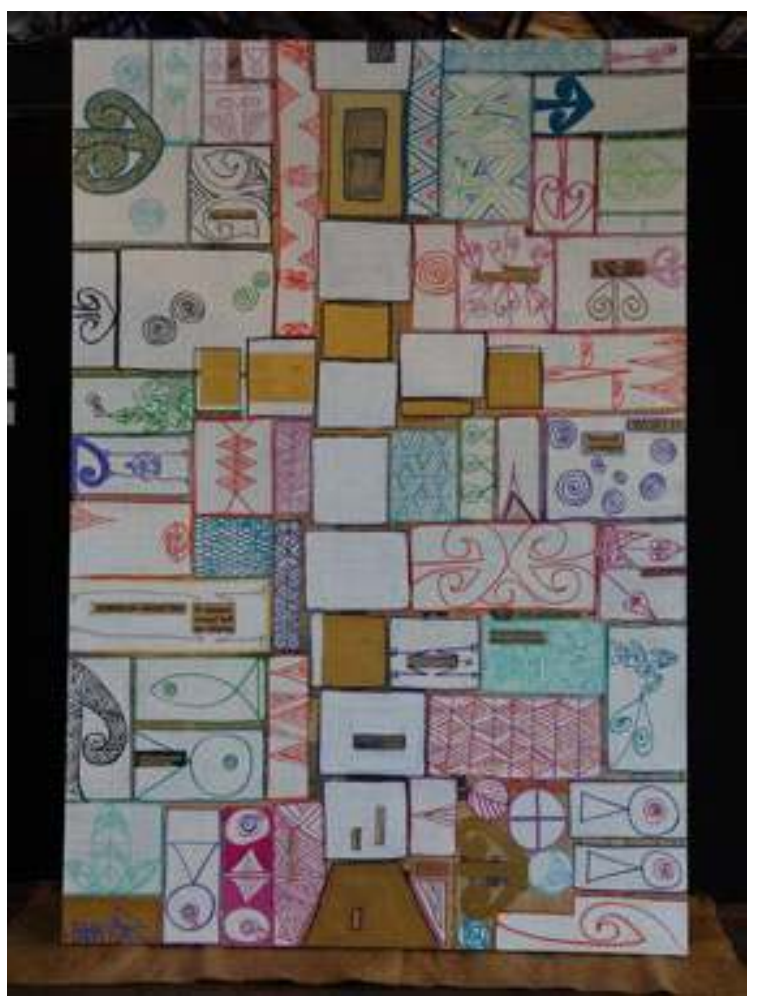

Tracey Tawhiao, date inconnue, peinture acrylique et pastel à l'huile sur journaux collés sur toile. Exposé dans le cadre du festival «Atamira Maori in the City », Auckland

(c) C. Pellini, 5 juin 2013

(fig. 4) Tracey Tawhiao est une artiste qui a suivi une formation universitaire en droit et a obtenu un diplôme dans ce domaine avant de se tourner vers la poésie puis la peinture lorsqu'elle a eu des enfants. Il s'agit là d'une caractéristique partagée par d'autres artistes. Elles sont nombreuses à s'être tournées vers une activité créative au moment où elles sont devenues mères. C'est aussi souvent à cette période qu'elles ont véritablement commencé à s'intéresser à la culture, à l'histoire et à la langue māori pour pouvoir transmettre à leurs enfants des connaissances auxquelles parfois elles n'avaient pas eu accès elles-mêmes car leurs parents voulaient qu'elles s'intègrent dans la société pākehā sans subir les discriminations qu'ils avaient pu connaître à cause de leur appartenance à la société māori. Tracey Tawhiao avait le sentiment que le droit néo-zélandais était toujours injuste envers les Māori et que la pratique artistique était alors plus adaptée qu'une carrière dans le domaine judiciaire ou législatif pour faire entendre les voix des Māori et ses opinions sur les questions relatives au territoire, à la culture, à la langue et à la spiritualité māori (entretien avec Tracey Tawhiao, le 13 novembre 2013). Certaines de ses œuvres dénoncent notamment la confiscation des terres, une thématique importante pour les Māori qui entretiennent des liens très forts avec le territoire ${ }^{8}$. Ce sujet est également traité par d'autres artistes comme Charlotte Graham qui évoque dans certaines de ses productions le traité de Waitangi sur lequel 
s'appuient encore aujourd'hui les Māori dans leurs démarches de revendications territoriales et leurs demandes de réparation des injustices perpétrées par le système colonial (à ce sujet, voir Orange ibid.) (fig. 5).

\section{T Triti}

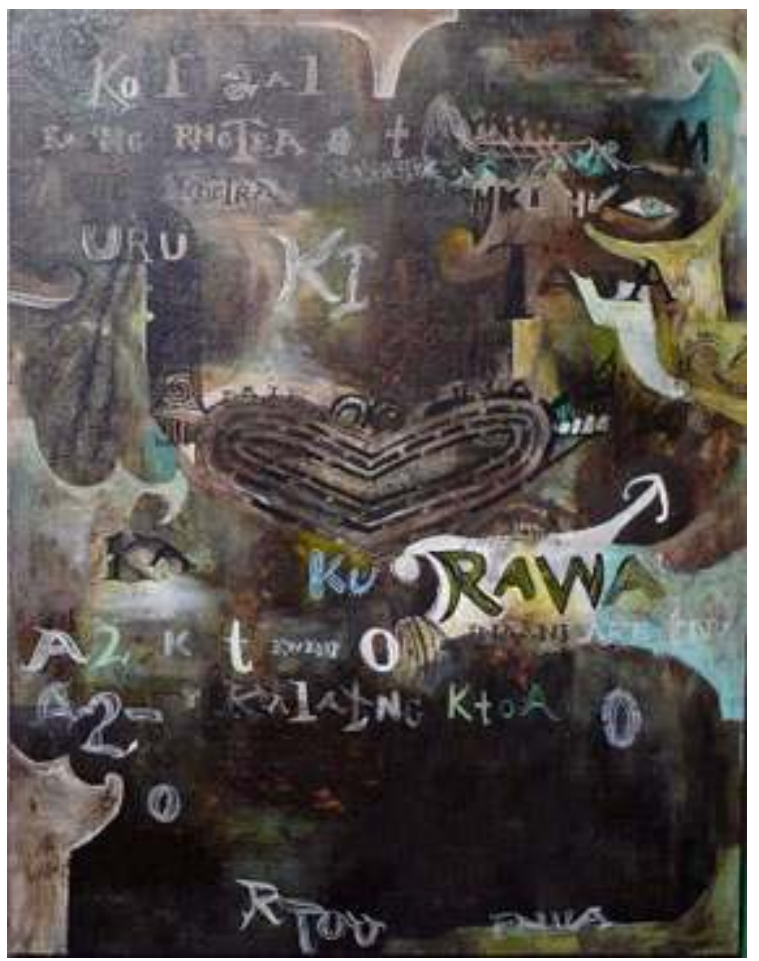

Charlotte Graham, 2009, peinture à l'huile et bitume sur toile, 710 x 560 mm, (http:// www.johnleechgallery.co.nz/artists/represented/charlottegraham/default.asp?artwork=1104). John Leech Gallery

5 Tracey Tawhiao utilise des pastels, des feutres peinture ainsi que de la peinture acrylique. Ces outils ont été introduits en Nouvelle-Zélande consécutivement à la colonisation; aujourd'hui de nombreuses artistes māori les emploient pour créer des œuvres aux styles très variés (fig. 6), (fig. 7), (fig. 8). 
Kia Kaha

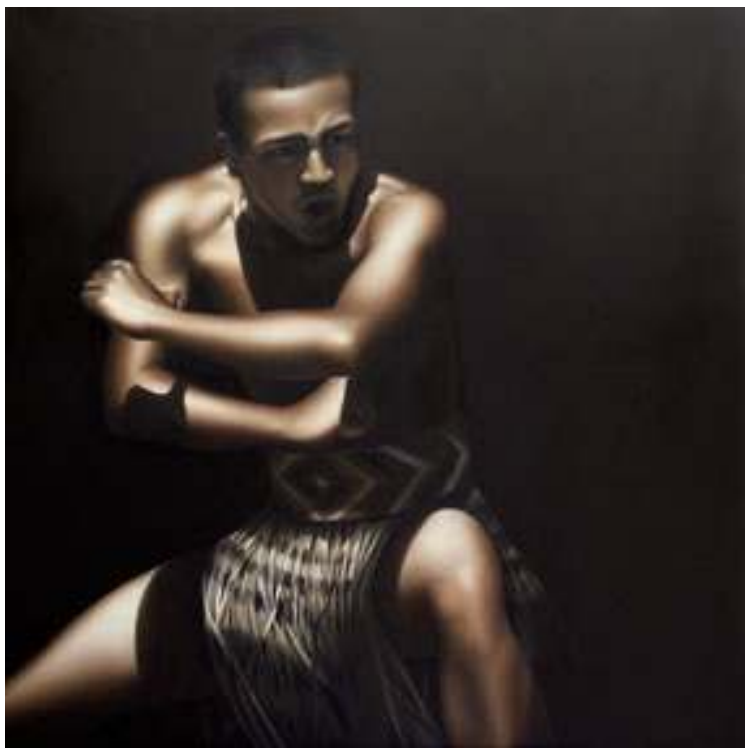

Sofia Minson, 2006, huile sur toile, $1300 \times 1300 \mathrm{~mm}$

http://www.newzealandartwork.com/product/kia-kaha

\section{Tidal flow}

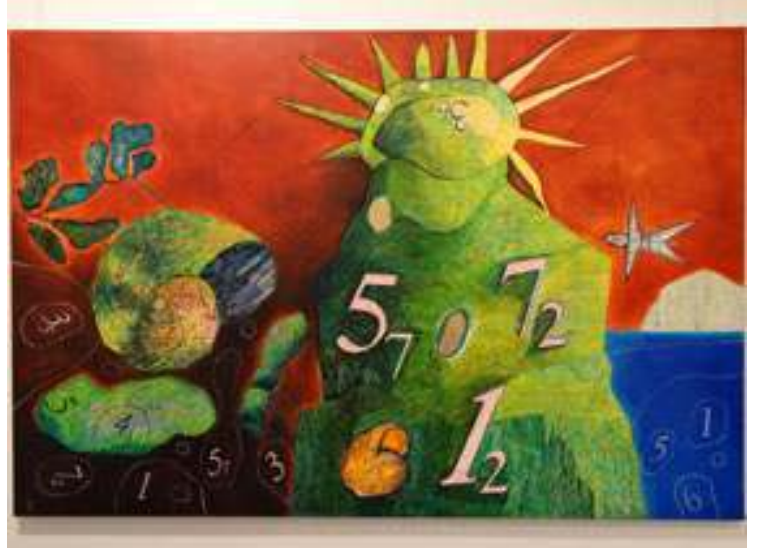

Mere Clifford, 2013, huile sur toile, $1015 \times 1520$ mm. Extrait de l'exposition « Ask that Mountain Puketapapa ", Nathan Homestead, Auckland

(c) C. Pellini, 12 mai 2013 


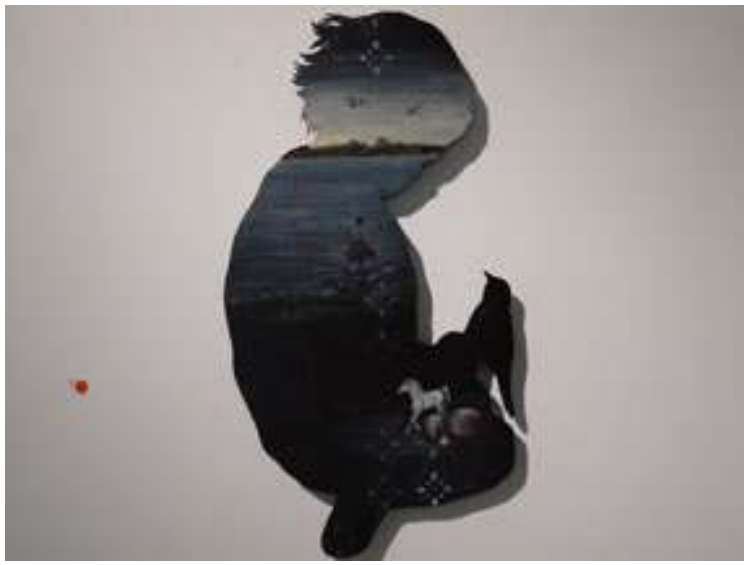

Penny Howard, 2013, peinture à l'acrylique sur panneau de fibre à densité moyenne, 600 x $330 \mathrm{~mm}$ Extrait de l'exposition « Te Kuri O Te Wao - You leave footprints in the soft earth of my heart », Whitespace, Auckland

(C) C. Pellini, 8 novembre 2013

6 Tracey Tawhiao affirme ne pas se soucier du fait que ses matériaux de prédilection ont été introduits par les Pākehā, ce qui est important pour elle est le message qu'elle véhicule et qui fait de ses œuvres de l'art māori (entretien du 13 novembre 2013). Outre des dessins de poissons rappelant l'environnement insulaire dans lequel ont vécu ses grands-parents māori, ses créations regorgent de motifs évoquant ceux présents dans les productions traditionnelles. Elle s'inspire particulièrement des kowhaiwhai ${ }^{9}$ et des koru utilisés en sculpture et dans les peintures ornant les maisons de réunion ${ }^{10}$ (fig. 9), (fig. 10).

\section{Kowhaiwhai}

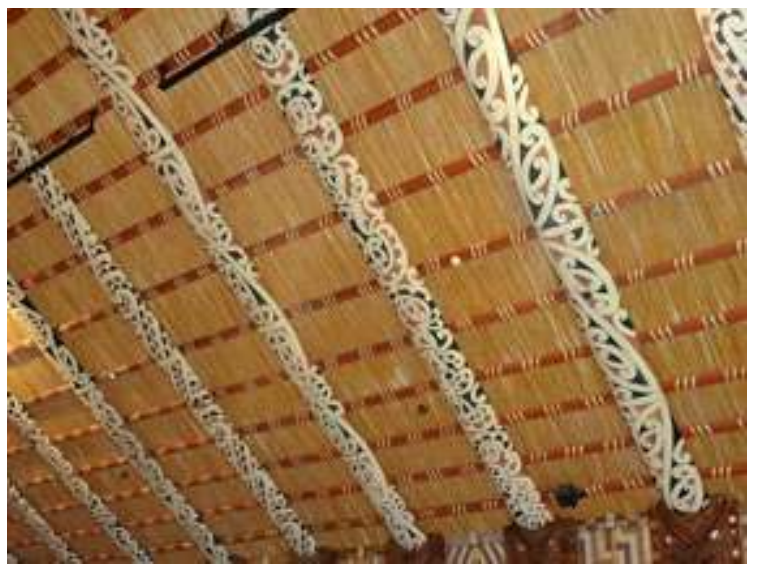

Intérieur d'une whare whakairo. Waitangi (c) C. Pellini, 5 juin 2013 
Loved (3)

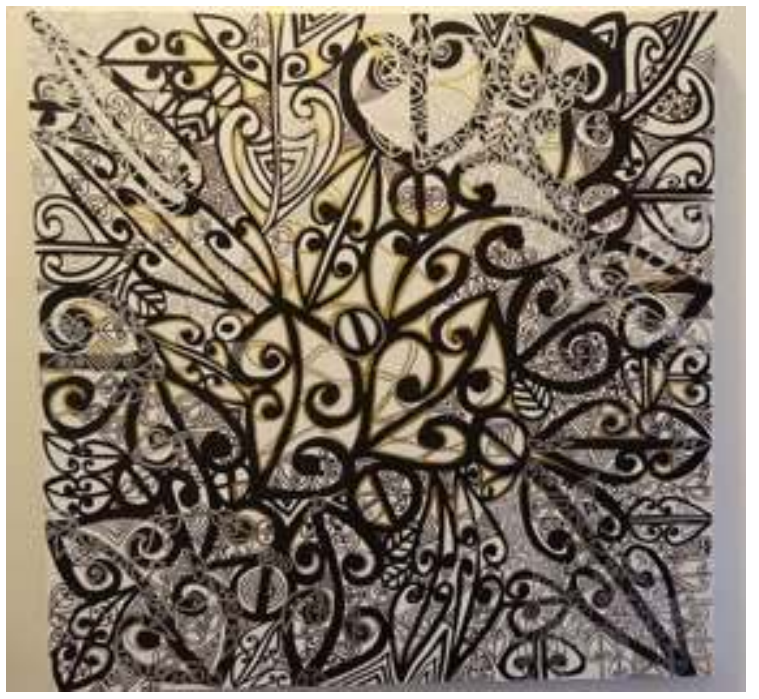

Tracey Tawhiao, date inconnue, peinture acrylique sur toile. Extrait de l'exposition « In Love », Auckland (c) C. Pellini, 17 juillet 2013

7 L'artiste réagence ces motifs d'une manière qu'elle considère pertinente et adaptée au contexte contemporain (op. cit.). Certaines de ses œuvres sont décoratives: elle peint des motifs de koru et de korowai sur des objets tels que des miroirs, des dessous de verre et d'assiette ou des plateaux (fig. 11), (fig. 12).

Sous-verres décorés par Tracey Tawhiao

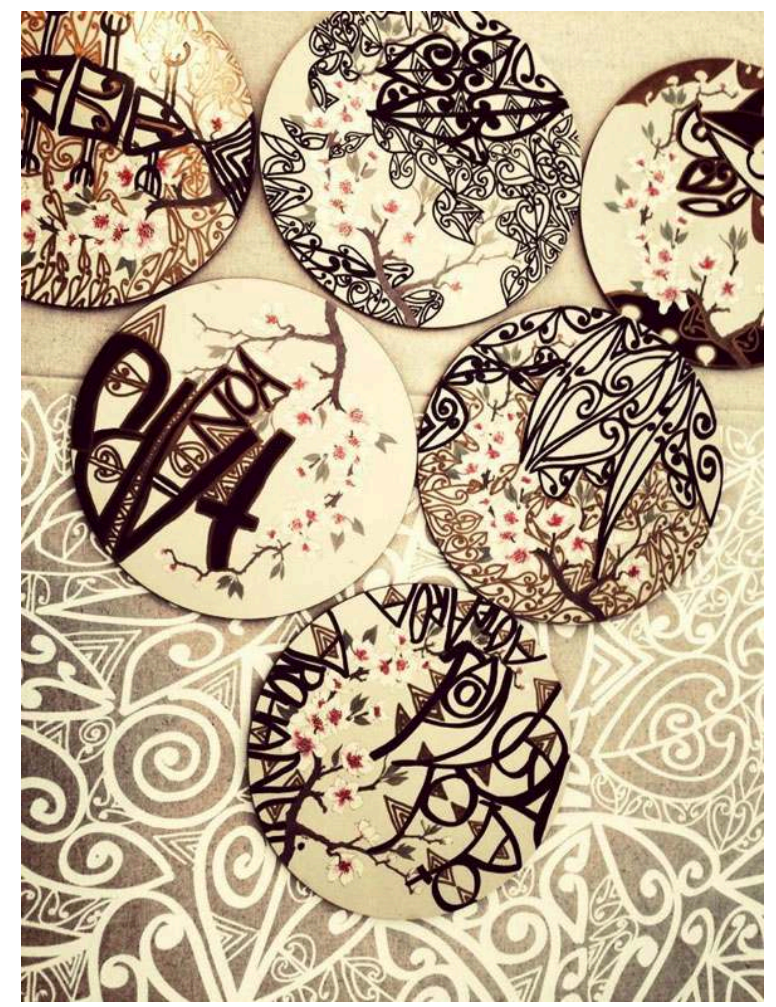

Photographie publiée sur le Facebook de l'artiste le 11 novembre 2014

(c) Tracey Tawhiao 
Miroir décoré au feutre peinture permanent

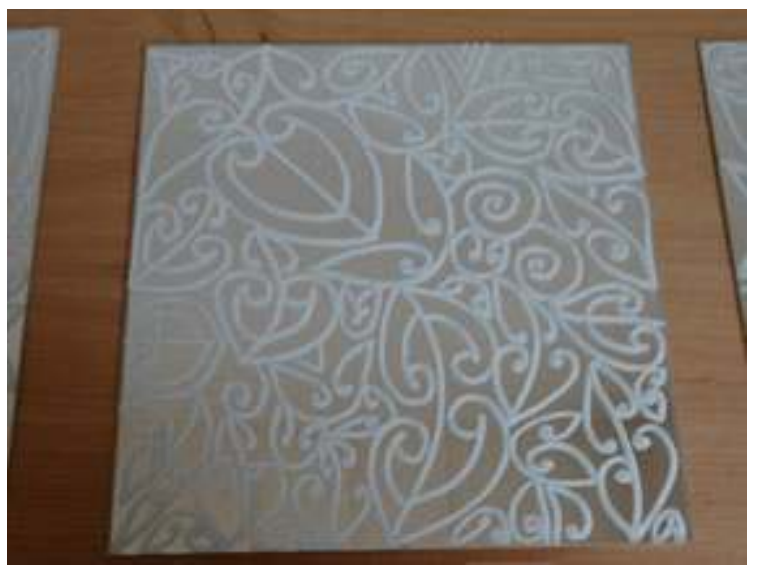

Tracey Tawhiao. Extrait de l'exposition «In Love», Ponsonby, Auckland (c) C. Pellini, 17 juillet 2013

D'autres témoignent de son indignation contre la politique appliquée en NouvelleZélande à l'encontre des Māori, par le passé ou de nos jours. Pour ce faire, Tracey Tawhiao aime se servir de journaux d'information néo-zélandais qu'elle recouvre d'aplats de couleurs et de motifs ne laissant apparaitre que quelques mots, auxquels elle ajoute parfois des lettres ou des termes supplémentaires (fig. 13).

\section{Euvre sur papier journal de Tracey Tawhiao}
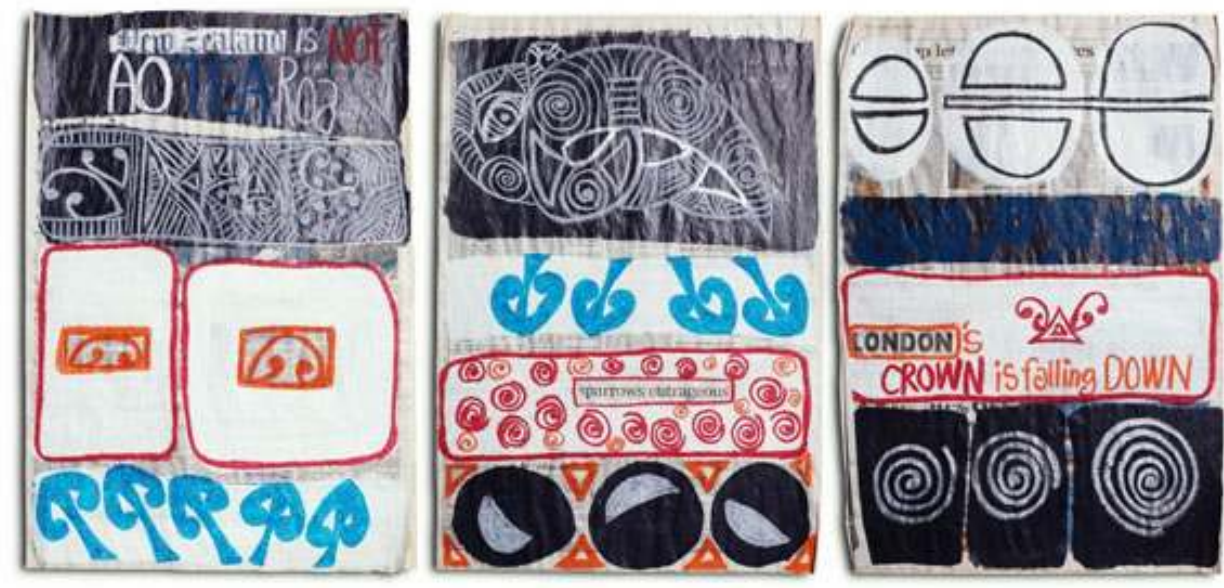

Présentée sur le blog internet de l'artiste « Art \& Poetry by Tracey Tawhiao

http://www.traceytawhiao.com/2010/08/

Elle souhaite ainsi subvertir le message de ces journaux symbolisant pour elle l'instauration de la société coloniale (op. cit.). Elle se sert de journaux écrits en anglais pour dénoncer les méfaits de la colonisation et elle utilise également la langue anglaise pour ses poèmes. Ses œuvres reflètent ainsi sa double culture : il s'agit d'une artiste māori ayant grandi et vécu dans un environnement urbain pākehā au sein duquel elle est désormais très active. En effet, elle a œuvré à la création de réseaux māori urbains majoritairement composés d'artistes māori (artistes visuels, chanteurs et acteurs) et de personnes s'intéressant aux milieux artistique et culturel ${ }^{11}$. Elle a ainsi fondé, en 2000 , 
le collectif House of Taonga ${ }^{12}$ qui regroupe des artistes māori visuels et d'autres œuvrant dans le domaine de la musique. Tracey Tawhiao organise des «Salons » qui offrent, aux artistes faisant partie de ce collectif ainsi qu'à d'autres qu'elle apprécie, l'occasion de se rencontrer et d'exposer leurs réalisations (fig. 14).

Figure 14

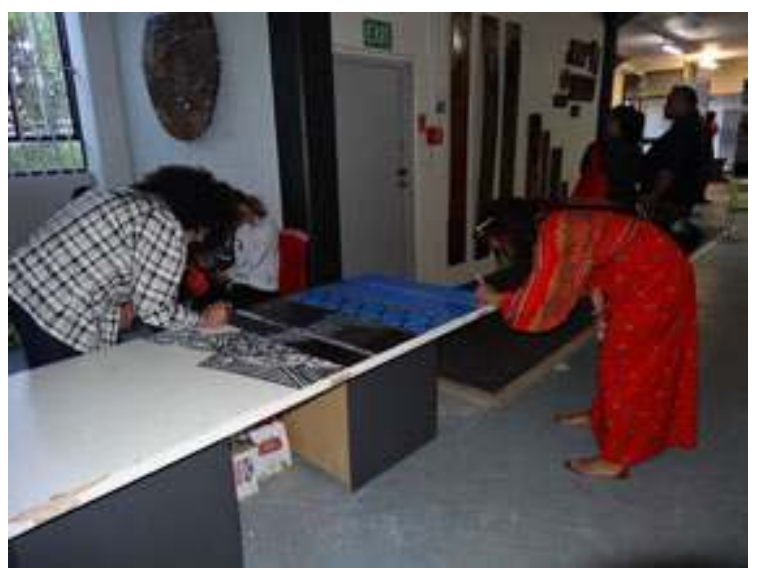

Tracey Tawhiao et Charlotte Graham en train de peindre ensemble sur une vieille porte durant le Salon de la House of Taonga « An art salon of us all », Auckland

(c) C. Pellini, 18 octobre 2013

Elle-même présente ses créations durant ces événements. Outre ces salons, Tracey Tawhiao expose souvent dans des lieux prêtés par des amis et des connaissances. Ce n'est que depuis peu qu'elle est reconnue par les institutions artistiques officielles néozélandaises. Artiste autodidacte, elle a auparavant essuyé de nombreuses critiques à propos de sa technique, notamment après que l'une de ses œuvres a été choisie pour figurer en couverture du livre Taiāwhio: Conversations with Contemporary Māori Artists (Smith 2002) (fig. 15). 


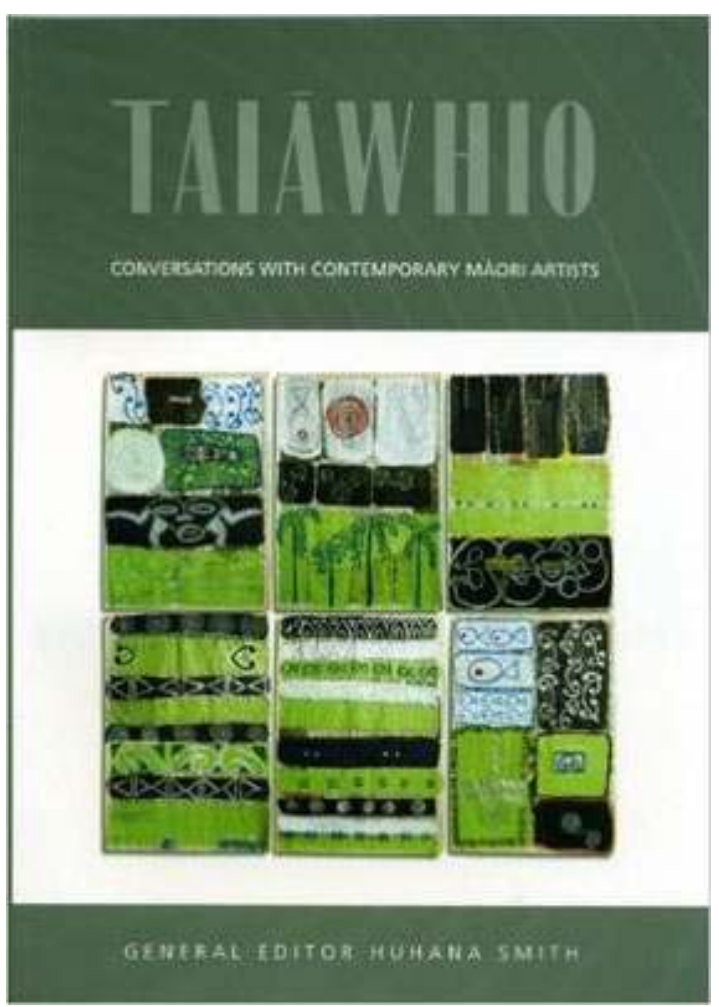

Conversations with Contemporary Māori Artists (Smith 2002), Te Papa Press

http://www.tepapa.govt.nz/TePapaPress/FullCatalogue/Art/Pages/Taiawhio.aspx

11 L'ouvrage a été publié par la maison d'édition du musée national Te Papa Tongarewa mais le musée n'a acheté aucune de ses productions. Le cas de Tracey Tawhiao n'est pas isolé. Lorsque j'ai visité le Te Papa en 2013, aucune œuvre de femmes māori ne faisait partie de la collection permanente consacrée à l'art māori contemporain. Quelques créations de Robyn Kahukiwa ${ }^{13}$ étaient exposées mais uniquement à l'étage avec les créations māori traditionnelles. Les œuvres des femmes māori sont plus nombreuses dans des galeries d'art importantes comme l'Auckland Art Gallery mais elles sont toujours minoritaires par rapport aux œuvres masculines. Pourtant, les femmes artistes māori sont très productives et elles sont souvent aussi présentes que les hommes, si ce n'est plus, lors des expositions organisées dans le cadre de festivals mettant la culture māori à l'honneur ou au sein d'expositions collectives consacrées à l'art māori contemporain. Plusieurs artistes parviennent cependant à exposer seules en dehors de ces moments phares, en Nouvelle-Zélande et à l'étranger. Tracey Tawhiao a par exemple présenté ses œuvres dans différents pays dont la France où elle a souvent collaboré avec George Nuku ${ }^{14}$ (fig. 16). 


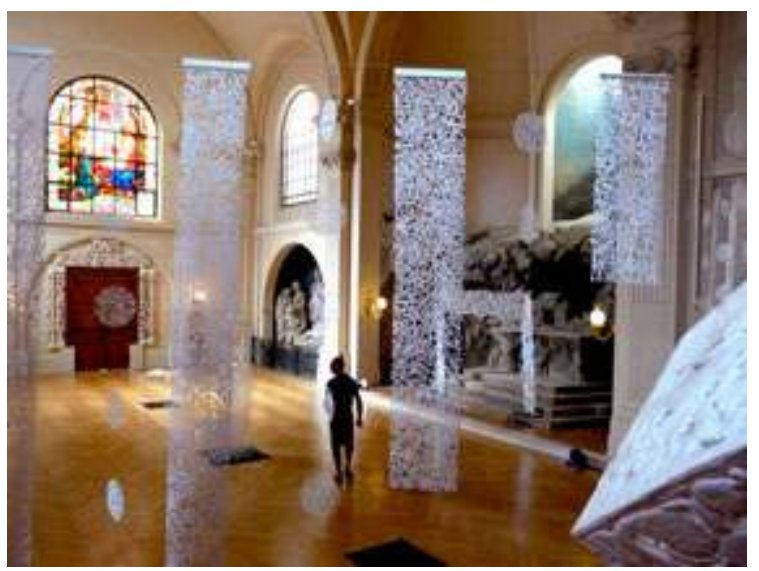

Euvres peintes de Tracey Tawhiao et œuvres sculptées par George Nuku, chapelle du Calvaire de l'église Saint-Roch, Paris, juin à septembre 2012

photographie issue du site internet de la House of Taonga à l'adresse http:// www.houseoftaonga.com/paris-exhibitions/

12 Elle dit aimer collaborer avec des hommes car elle trouve qu'un équilibre s'établit ainsi entre son énergie créative féminine et celle procurée par les hommes. Cette notion d'équilibre qui revient souvent dans les discours des artistes est très importante dans la culture māori (Patterson 1992). Tracey Tawhiao y est attachée et la philosophie māori nourrit sa pratique artistique novatrice déployée dans un environnement urbain contemporain.

\section{L'argile comme substitut à la sculpture sur bois : les œuvres « sculptées » d'Hera Johns}

On retrouve une idée similaire chez Hera Johns, une artiste qui s'inspire de la culture sur bois traditionnelle māori mais en présente une relecture originale. Elle ne travaille pas le bois mais «sculpte " l'argile en traçant sur cette matière non cuite des motifs assez similaires à ceux ornant traditionnellement les objets en bois sculptés (fig. 17).

\section{Kumara Hou 1"}

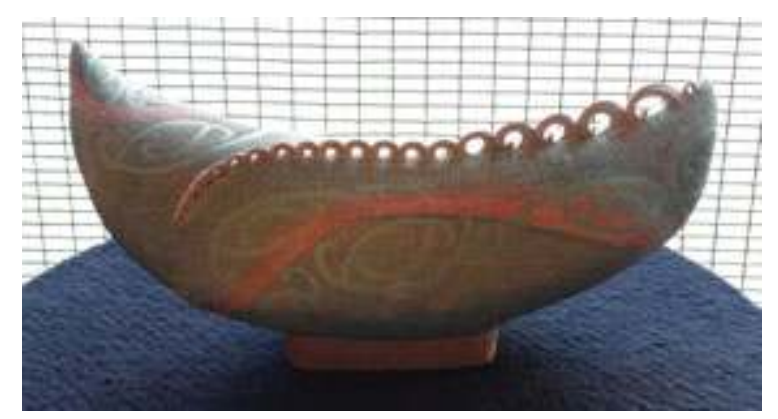

Hera Johns, 2013, argile blanche, engobe coloré, 350 × 110 mm. Exposé dans le cadre du festival "Atamira Maori in the City », Auckland

(C) C. Pellini, 7 septembre 2013

13 Hera Johns est elle aussi une artiste autodidacte qui a seulement bénéficié d'une formation artistique peu poussée durant sa scolarité et des conseils de Manos Nathan, 
un artiste māori reconnu, au cours d'ateliers. Elle a commencé en sculptant des pierres et des os grâce à une machine présente à son domicile car confectionnée par son père à partir des pièces de plusieurs machines endommagées. Quand elle a quitté son milieu familial rural pour s'installer à Auckland, elle a dû changer de technique et elle s'est alors tournée vers l'argile. Elle a choisi ce matériau pour des raisons pratiques car il est peu coûteux et ne présente pas de dangers de toxicité pour ses enfants, mais aussi pour son aspect symbolique. En effet, utiliser de l'argile peut permettre de faire référence à Papatūānuku, la Terre Mère. L'argile renvoie également à l'élément féminin car dans certains récits mythiques, la première femme, Hine-ahu-one, a été créée par Tane, une divinité masculine, à partir de l'argile rouge nommée kōkōwai et se trouvant au niveau du pubis de Papatūānuku (Melbourne 2011 : 105-106; Hibbs 2006 : 4 ; Pihama 2001 : 17). Il s'agit donc d'un élément important dans la culture māori mais il n'existe pas de tradition māori de poterie. La pratique artistique d'Hera Johns se distingue donc de l'art traditionnel. Cette artiste aime introduire de la couleur dans ses œuvres mais sans utiliser de vernis. Elle utilise alors la technique de l'engobe : elle délaie des pigments colorés dans une fine couche d'argile qu'elle applique sur le corps de l'œuvre avant cuisson. Elle produit ainsi des pièces colorées présentant des dégradés de couleurs (fig. 18).

Pendentifs réalisés par Hera Johns

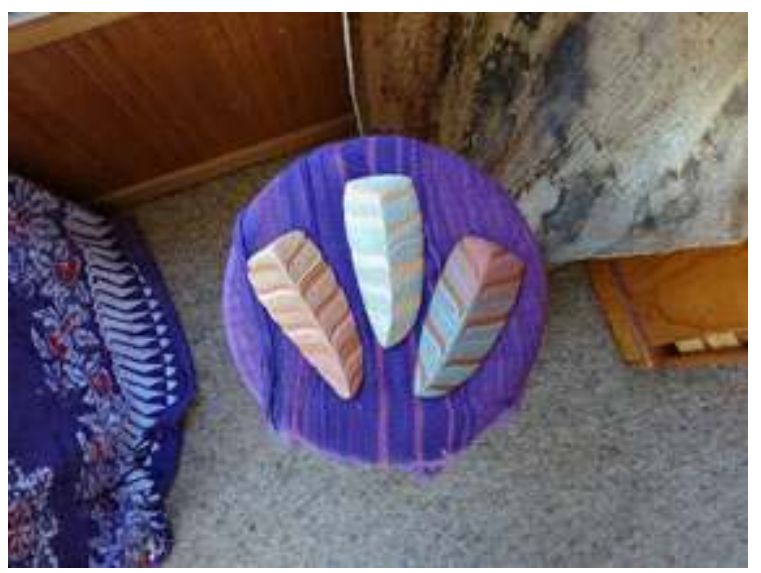

Argile et engobe coloré, Birkenhead, Auckland

(c) C. Pellini, au domicile de l'artiste, 3 septembre 2013

Toujours avant de cuire l'argile, elle la travaille au moyen d'outils de sculpteurs pour "sculpter» les plus gros motifs, et de limes à ongle, pour les plus fins. Ses tracés rappellent fortement les motifs ornant par exemple les tiki en bois, des personnages anthropomorphes sculptés. Elle crée ainsi des pendentifs et des œuvres aux formes variées faisant référence à des éléments culturels et historiques māori. Elle a, entre autres, réalisé une série de kumara en argile. Les kumara sont des patates douces qui représentaient une source de nourriture importante pour les Māori avant la colonisation. Elles revêtent aussi pour Hera Johns une signification personnelle car le chef d'une whānau (famille étendue) dont elle était très proche cultivait un champ de kumara et lui avait demandé il y a quelques années d'en faire des croquis. Elle n'avait pas osé car elle ne se sentait pas encore prête à obtenir un résultat satisfaisant. Ayant le sentiment d'avoir progressé, elle les a enfin réalisées en argile en 2013 (fig. 19). 
Hera Johns en train de « sculpter » de l'argile

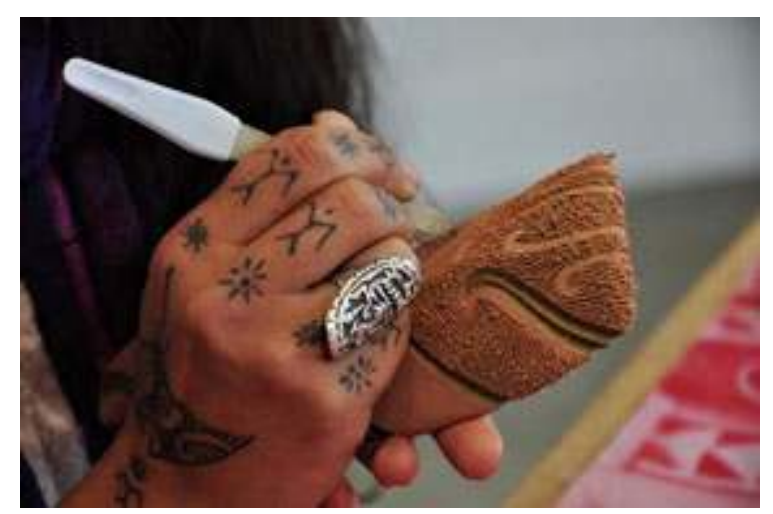

Au cours d'une démonstration à la Kura Gallery d'Auckland (c) C. Pellini, 11 juillet 2013

15 Le travail de l'argile permet à Hera Johns de ne pas transgresser le caractère tapu (sacré) de la sculpture qui est encore assez fort parmi les artistes māori puisque, comme nous l'avons déjà évoqué, les grandes productions sculptées pouvaient être seulement réalisées par des hommes. De nos jours, la sculpture sur bois n'est plus un domaine exclusivement réservé aux artistes masculins: lors de mon séjour en Nouvelle-Zélande, j'ai notamment eu l'occasion de voir une œuvre sculptée par une jeune femme. Certaines artistes avec lesquelles je me suis entretenue m'ont également dit que l'apprentissage de la sculpture n'était généralement plus interdit aux filles. Mais elles ont également précisé qu'il y avait une différence entre l'enseignement prodigué dans les établissements «à l'occidentale » et celui, traditionnel, dispensé par les experts en sculpture sur bois. Contrairement au premier, ce dernier est plus attaché aux valeurs et aux savoirs traditionnellement associés à la sculpture et il est donc plus difficile de trouver des maîtres sculpteurs opérant dans ce contexte, qui acceptent de prendre des femmes pour élèves. Les artistes interrogées connaissent des femmes s'étant engagées dans cette voie et elles les admirent car elles ont dû souvent lutter pour être acceptées mais elles-mêmes ne se sont pas tournées vers la sculpture, soit parce que cette pratique ne les a jamais vraiment attirées, soit parce que selon leurs propres termes elles ne se sentent "pas confortables " ${ }^{15}$ à cette idée. Elles auraient l'impression de briser un tapu alors qu'elles considèrent qu'il est très important de faire preuve de respect envers la culture māori. Cependant, la sculpture sur bois représentant une part conséquente de l'art traditionnel māori, elle est pour nombre d'entre elles une véritable source d'inspiration. Certaines artistes ont alors développé des stratégies pour faire référence aux productions sculptées traditionnelles sans pour autant sculpter le bois. Les artistes qui privilégient la peinture peuvent insérer dans leurs productions peintes des motifs appartenant au répertoire de la sculpture māori ou même représenter des éléments traditionnellement sculptés comme les tiki (fig. 20). 
Turangawaewae

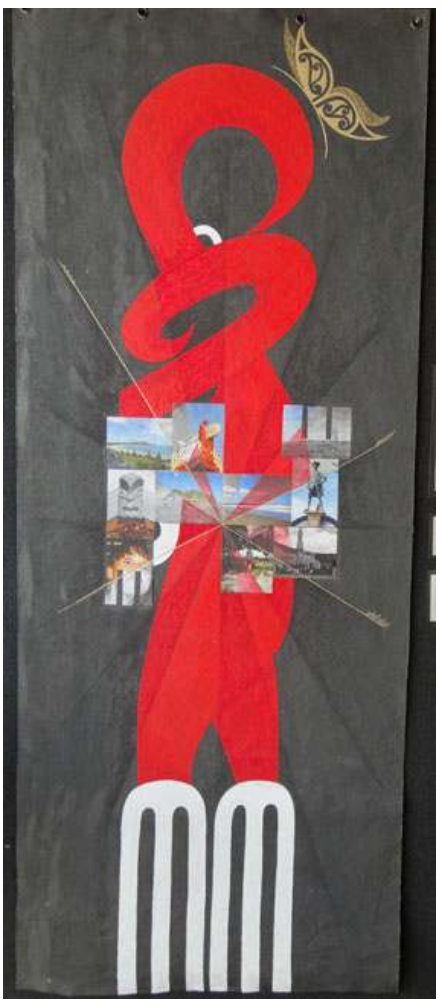

Terangi Kutia-Tataurangi, 2013, peinture acrylique, pastel et montage photographique sur toile non tendue. Exposé dans le cadre du festival « Atamira Maori in the City », Auckland (C) C. Pellini, 7 septembre 2013

Des artistes comme Natasha Keating utilisent des morceaux de bois non pas pour les sculpter mais comme support pour leurs créations peintes ou dessinées (fig. 21). 


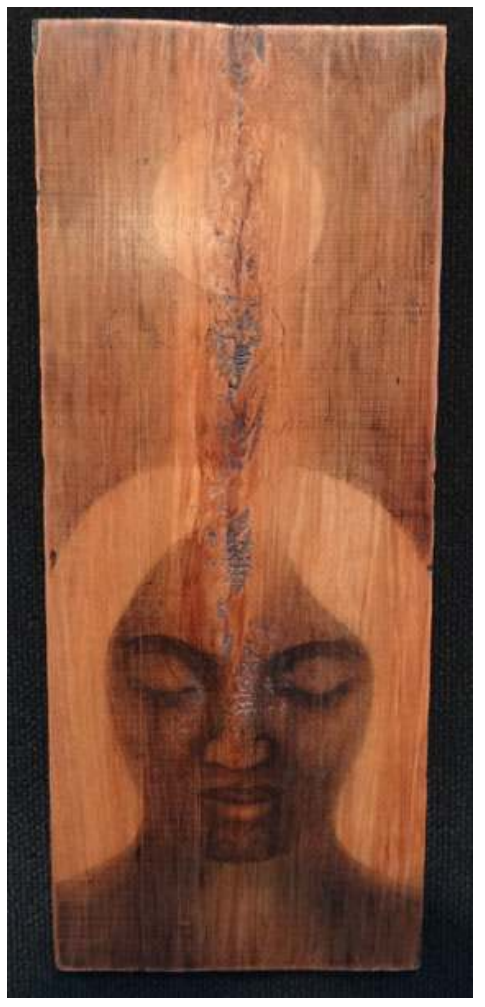

Natasha Keating, 2013, teinture pour bois et crayon sur bois de rimu recyclé. Exposé dans le cadre du festival "Atamira Maori in the City », Auckland

(C) C. Pellini, 7 septembre 2013

D'autres, à l'instar d'Hera Johns, se sont aussi tournées vers l'argile. Jane Matua, par exemple, fait référence au bois en expérimentant diverses techniques de cuisson de l'argile afin de conférer à ses œuvres un aspect similaire à celui du bois (fig. 22).

\section{Material Culture Artefacts}

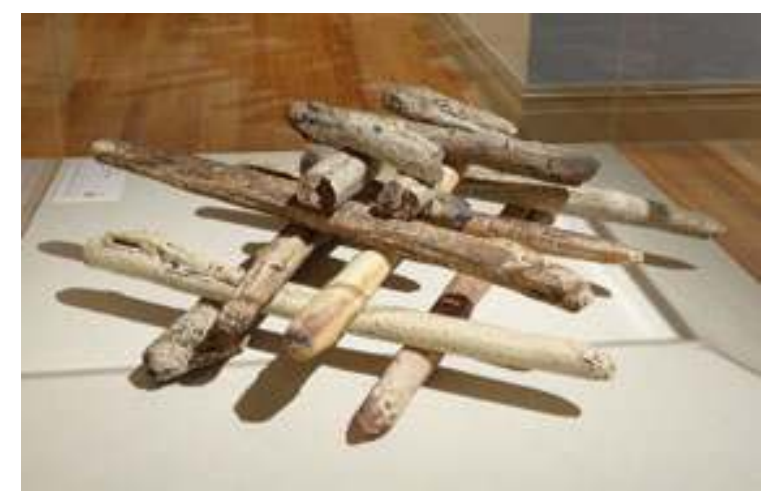

Jane Johnson-Matua, 2013, argile de Rotorua. Exposé au Rotorua Museum

(C) C. Pellini, 26 octobre 2013

Dans le cas d'Hera Johns, utiliser de l'argile lui permet de faire référence à l'art traditionnel tout en innovant et c'est aussi pour elle un moyen d'initier un dialogue sur l'importance de Papatūānuku, la Terre-Mère et sur la nécessité de protéger l'environnement. Elle participe à des ateliers auprès d'adultes et d'enfants à qui elle 
transmet son savoir-faire. Elle fait également partie d'un collectif d'artistes spécialisés dans le travail de l'argile, Nga Kaihanga Uku - The Maori Clayworkers, et elle participe à des expositions collectives. Hera Johns a acquis une certaine renommée puisqu'elle a été sélectionnée pour représenter la Nouvelle-Zélande en compagnie d'autres artistes māori au XI ${ }^{e}$ Festival des arts du Pacifique aux Îles Salomon. Mais sa pratique artistique ne lui permet pas encore de gagner correctement sa vie et lorsque nous nous sommes rencontrées elle était à la recherche d'une activité rémunérée (entretien du 3 septembre 2013). C'est également le cas de nombreuses artistes: quelques-unes arrivent à vivre de leur art mais la plupart ont un emploi complémentaire. Elles doivent alors concilier leur pratique artistique, leur travail rémunéré et leur vie familiale. Toutes n'arrivent pas à trouver un emploi en lien avec l'art mais c'était le cas de l'une des artistes rencontrées durant mon enquête de terrain : Vicky Thomas.

\section{Photographie et problématiques identitaires : l'exemple de Vicky Thomas}

Vicky Thomas est une artiste photographe qui est aussi la gérante de la galerie d'art Kura Gallery située au centre d'Auckland. Kura Gallery expose et propose à la vente des œuvres réalisées en grande majorité par des artistes māori. Cet emploi permet à Vicky Thomas de présenter certaines de ses œuvres dans cette galerie et sur le site internet de cette dernière. Le fait d'être gérante lui offre aussi l'opportunité d'être en contact avec des artistes māori très différents : des hommes, des femmes, des artistes ruraux et d'autres urbains créant des peintures, des sculptures, des bijoux, des objets décoratifs ou encore du mobilier design. Il s'agit donc d'une expérience enrichissante qui nourrit sa propre pratique a priori très éloignée de l'art traditionnel puisque Vicky Thomas prend des photographies numériques qu'elle retouche grâce à des logiciels, puis imprime (fig. 23).

Te pahutanga te puawaitanga - Lava Lava

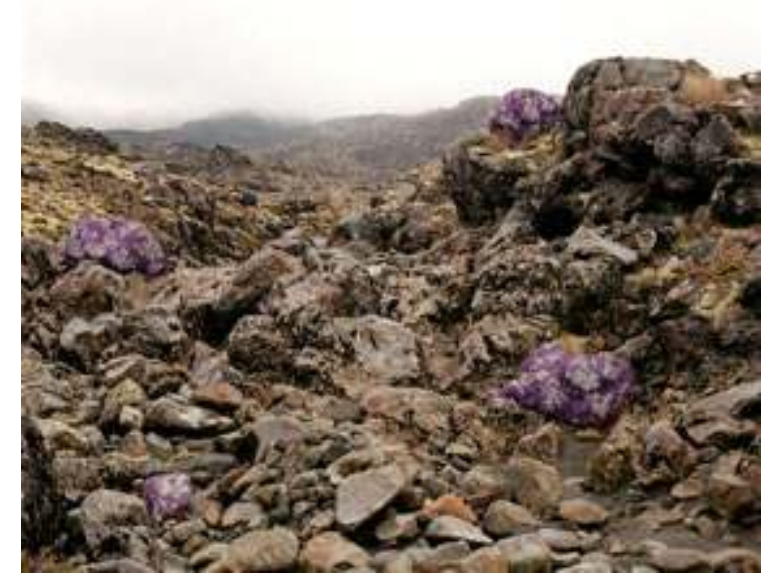

Vicky Thomas, 2005, impression photographique LED, 600 x 500 mm, Kura Gallery http://www.kuragallery.co.nz/vicky-thomas-9

L'utilisation de ces nouveaux médias lui permet d'explorer des questionnements identitaires et culturels. Ainsi, elle a réalisé une série d'autoportraits qui fait écho en s'y opposant aux pratiques de la photo coloniale et des portraits peints réalisés par des 
Pākehā présentant leurs visions des Māori. Avec ces autoportraits, Vicky Thomas se réapproprie sa perception personnelle de son image et expose la manière dont elle conçoit son identité en allant au-delà des étiquettes "Māori » et "Pākehā » et en insistant sur sa personnalité propre. Elle se représente ainsi sous de multiples facettes : des éléments tels que le bandeau avec des kowhaiwhai et le dessin semblable à un tatouage sur le menton renvoient à la culture māori, la peau maquillée en blanc et la perruque blonde de la deuxième photo évoquent une identité pākehā et le troisième portrait fait référence à des influences extérieures comme le mouvement de la « Blaxploitation » (fig. 24, 25, 26).

\section{Self Portrait \#1}

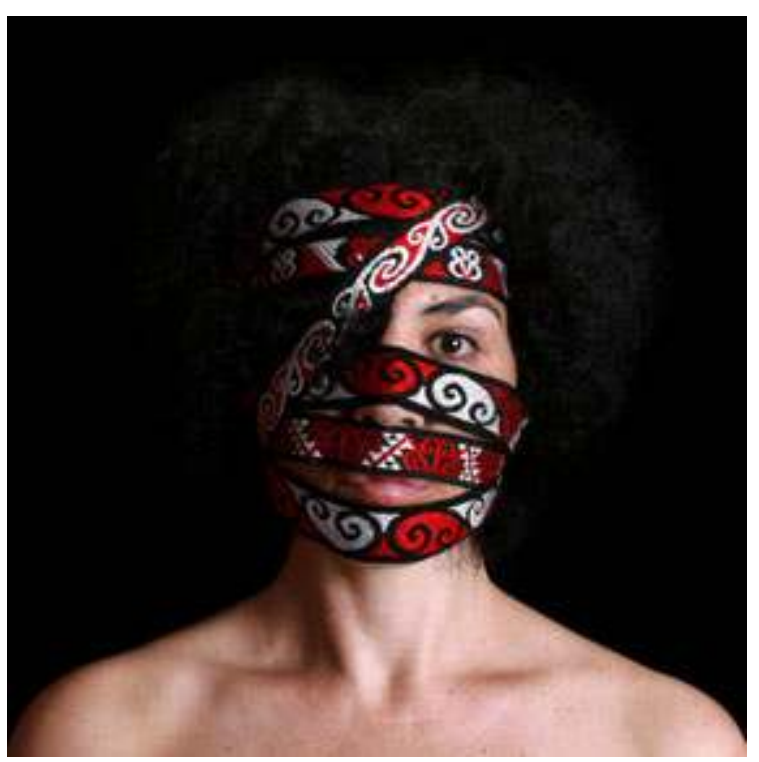

Vicky Thomas, 2009. Série « Self Portrait», Kura Gallery

http://www.kuragallery.co.nz/vicky-thomas-12 


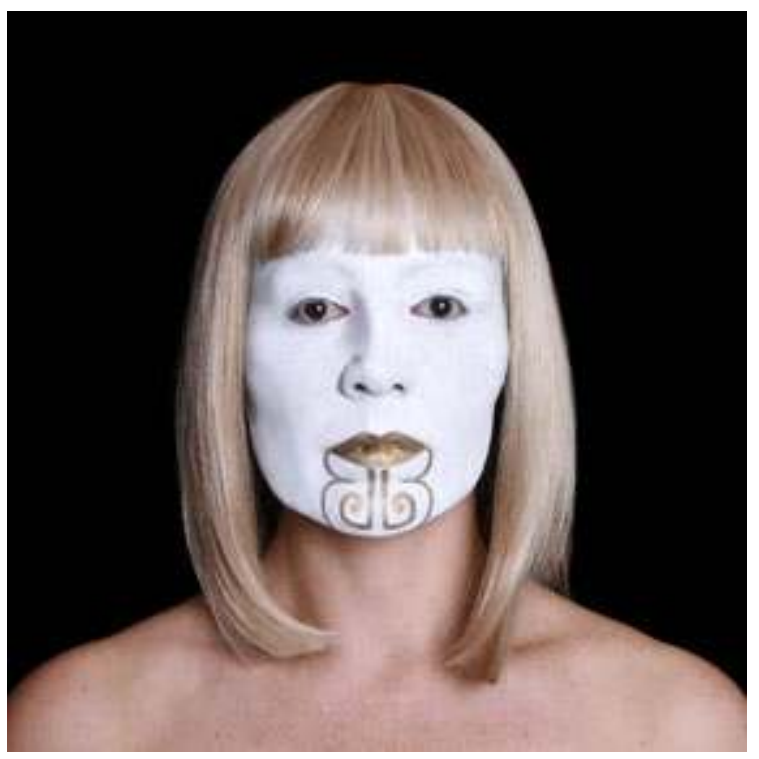

Vicky Thomas, 2009. Série « Self Portrait », Kura Gallery ( http://www.kuragallery.co.nz/vicky-thomas-13

\section{Self Portrait \#3}

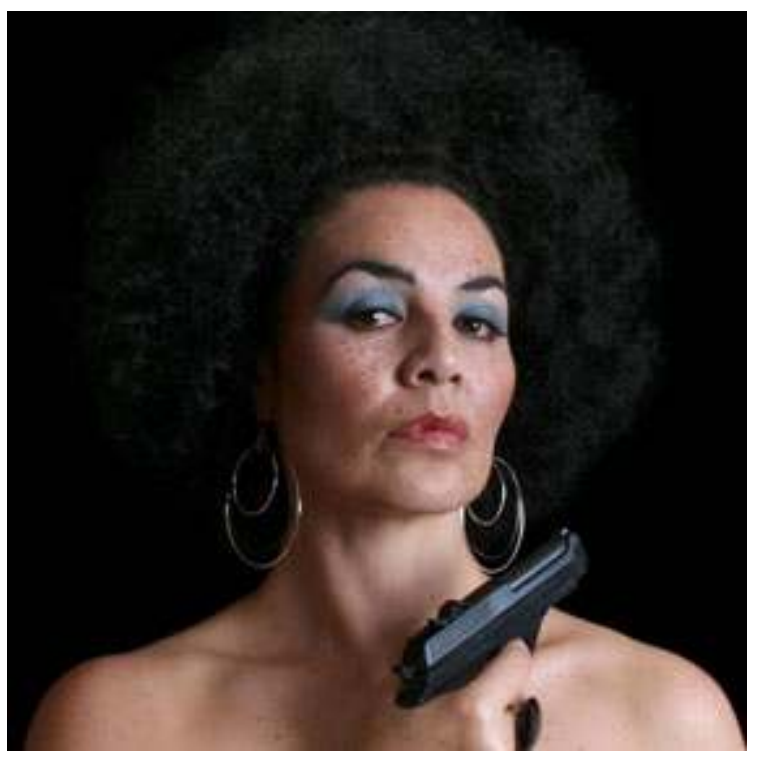

Vicky Thomas, 2009. Série « Self Portrait », Kura Gallery http://www.kuragallery.co.nz/vicky-thomas-14

19 Avec de telles œuvres, l'artiste semble mettre en exergue son genre, mais tel n'est pas son objectif. Elle souhaite évoquer son expérience de femme māori qui évolue dans un milieu urbain colonisé, et présenter la manière dont elle conçoit son identité. Elle est une femme; cet aspect constitutif figure naturellement dans ses créations. La série "Miss Appropriate» aborde un thème similaire. Ces photographies exposent une danseuse vêtue d'une jupe rouge recouverte d'une piupiu (une jupe courte māori traditionnelle réalisée en fibres végétales) et portant des chaussures noires à talons fins. La jupe rouge à l'occidentale et les escarpins contrastent avec la piupiu et avec le 
moko kauae (tatouage au menton) que porte la danseuse. Vicky Thomas offre ainsi l'image d'une femme affichant des éléments issus des mondes māori et pākehā. Elle souhaite montrer que les femmes māori peuvent très bien réussir dans un contexte européen (entretiens avec Vicky Thomas des 5 et 31 juillet 2013) (fig. 27), (fig. 28).

Poi III

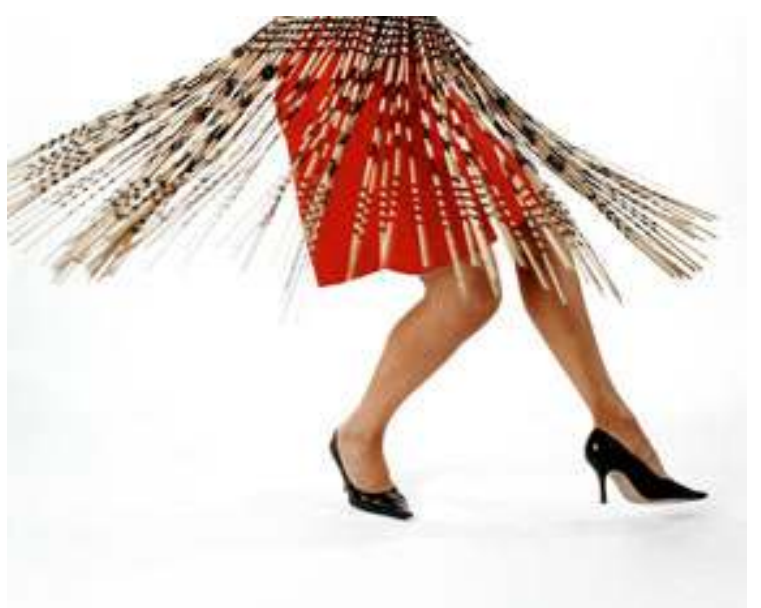

Vicky Thomas, 2004, impression photographique LED, 500 × 400 mm. Série « Miss Appropriate », Kura Gallery

http://www.kuragallery.co.nz/vicky-thomas-3

Miss Appropriate

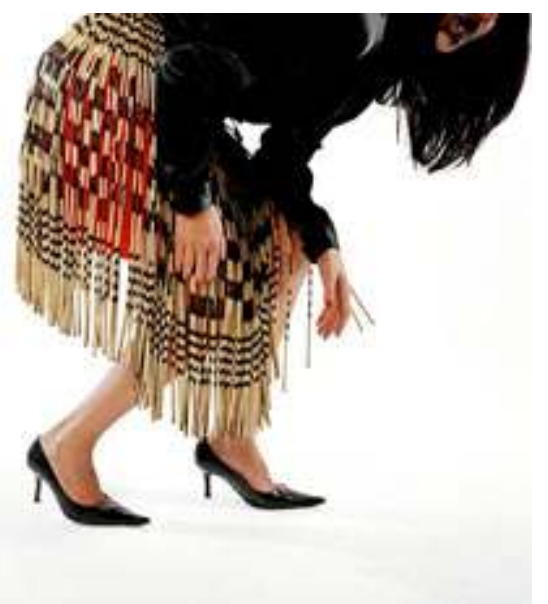

Vicky Thomas, 2004, Impression photographique LED, 500 x 400 mm. Série « Miss Appropriate », Kura Gallery

http://www.kuragallery.co.nz/vicky-thomas-3

L'artiste réalise aussi des photographies de paysages. Certains d'entre eux portent comme titres les noms māori correspondant aux lieux photographiés afin de montrer les liens qu'entretiennent les Māori avec leurs territoires. Vicky Thomas n'a pas ressenti le besoin d'inclure des motifs māori car pour elle ces lieux sont déjà intrinsèquement māori. Indiquer leur nom māori était alors suffisant pour véhiculer l'idée explorée ici (entretien du 5 juillet 2013) (fig. 29). 


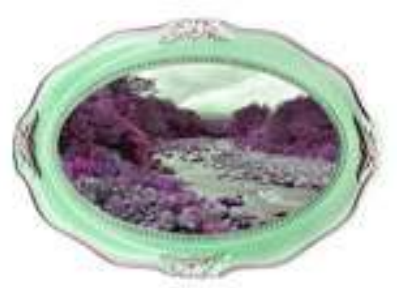

Vicky Thomas, impression sans cadre. Série « Rangi and Papa », Kura Gallery http://www.kuragallery.co.nz/vicky-thomas-19

D'autres photographies de paysages répondent à un objectif différent : l'artiste y insère des motifs de tapisserie et de textiles européens afin que les spectateurs pākehā trouvent un élément familier dans ses œuvres (fig. 30).

\section{He punawai he puawai - Rose water}

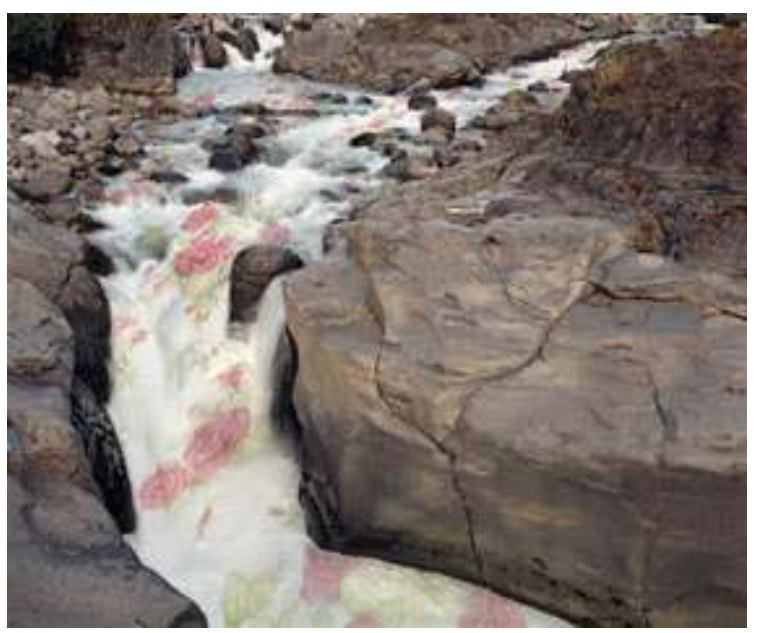

Vicky Thomas, 2005, impression photographique LED, 600 x 500 mm, Kura Gallery http://www.kuragallery.co.nz/vicky-thomas-6

Elle veut en effet les rendre accessibles à divers publics. C'est cette volonté qui l'a poussé à imprimer certaines de ses photographies sous la forme de posters peu onéreux. D'autres artistes comme Bronwyn Waipuka Callander - une artiste ayant d'abord pratiqué la peinture à l'huile et à l'acrylique mais qui se concentre désormais sur la réalisation d'illustrations et de dessins numériques - privilégient également les impressions pour pouvoir diffuser massivement leurs œuvres à un prix abordable (fig. 31). 
Mana Whenua : Taku Kai, Taku Oranga

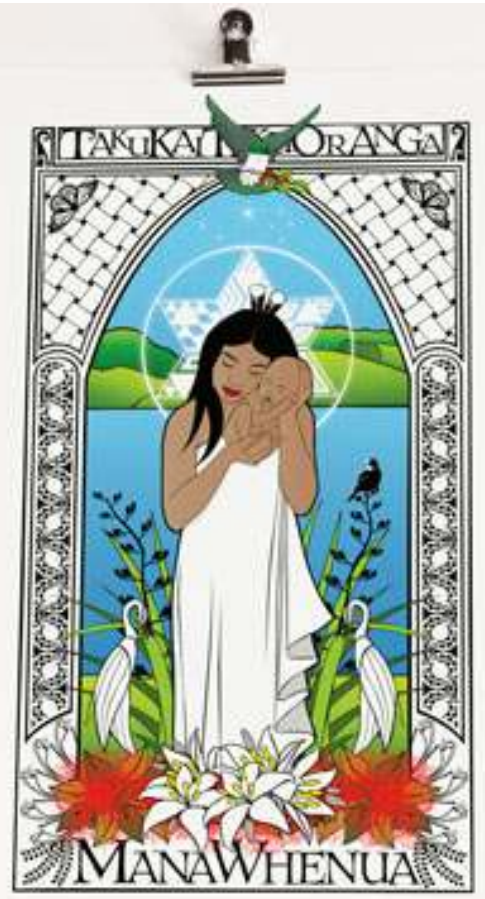

Bronwyn Waipuka-Callander, 2013, impression sur papier

(C) C. Pellini, atelier de l'artiste, 9 novembre 2013

23 Outre cette dimension, les photographies de Vicky Thomas révèlent une combinaison d'influences māori et pākehā. Son œuvre intitulée Indicating Navigators est particulièrement intéressante à ce sujet (fig. 32).

Indicating Navigators

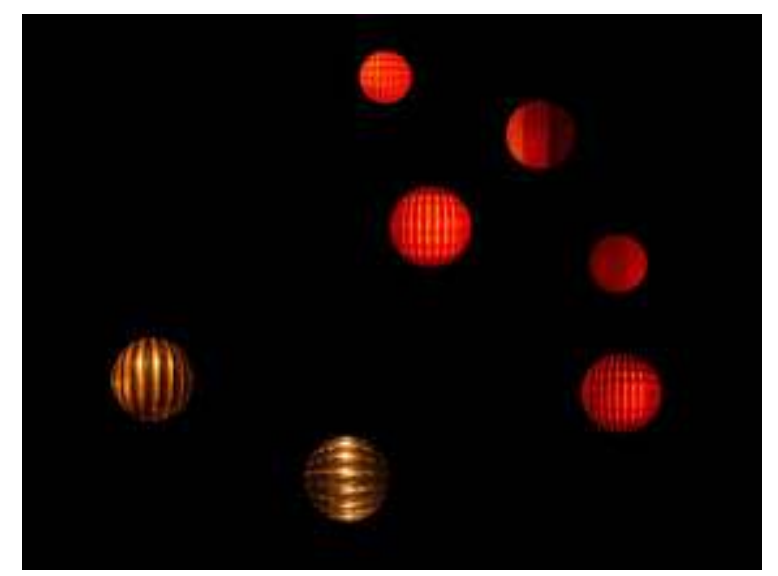

Vicky Thomas, 2009, impression encadrée, 1060 x 810 mm, Kura Gallery http://www.kuragallery.co.nz/vicky-thomas-11

Elle représente des sphères lumineuses agencées de la même manière que les étoiles composant la constellation Matariki dont l'apparition marque pour les Māori le début de la nouvelle année. Ces sphères sont en réalité des phares de voitures de la marque 
Subaru. Avec cette photographie, Vicky Thomas fait référence aux talents de navigation des Māori qui se dirigeaient grâce aux étoiles. L'artiste vit dans un contexte très différent de ce qu'était le milieu de vie des Māori qui se déplaçaient dans le passé par voie maritime avec des étoiles pour points de repère. Elle a alors voulu montrer qu'elle évolue dans un environnement urbain dans lequel elle "navigue " grâce aux panneaux de circulation et aux voitures. De plus, cette œuvre contient un jeu de mot dissimulé : Subaru désigne en japonais l'amas d'étoiles Matariki connu en français sous le nom de Pléiades. L'œuvre évoque alors les multiples connexions existant entre diverses cultures et le fait que la culture māori est toujours présente et vivante dans le monde contemporain dans lequel l'artiste évolue, même s'il paraît bien différent de celui qu'ont pu connaître les Māori dans le passé (op. cit.).

Vicky Thomas a commencé à créer ce genre d'œuvres au cours de ses études dans un établissement polytechnique, l'Unitec Institute of Technology d'Auckland. Sa formation a été très enrichissante mais elle a tout de même eu le sentiment qu'il s'agissait d'un environnement très peu adapté aux Māori. Elle a en effet dû affronter l'incompréhension de la majorité de ses professeurs ainsi que celle des autres étudiants face aux œuvres qu'elle créait pour exprimer son ressenti de jeune femme māori vivant dans un milieu urbain pākehā. Cela l'a poussé à développer les thèmes qu'elle explore aujourd'hui. Son expérience est similaire à celles vécues par d'autres artistes māori pour lesquelles le milieu universitaire s'est souvent avéré être un lieu où s'est éveillé leur intérêt pour des problématiques culturelles et identitaires. Mais, si la majorité des artistes rencontrées ont suivi des cursus universitaires dans des établissements «à l'occidentale », ce n'est pas le cas de la prochaine artiste que nous allons présenter.

\section{Les productions tissées de Deborah Duncan : entre tradition et modernité}

Deborah Duncan pratique une technique a priori très proche de l'art traditionnel māori : elle réalise des tissages. Elle a débuté en produisant des kete, des paniers tressés, qu'elle a appris à créer en intégrant un « weaving group ». Grâce à ce groupe, elle a pu bénéficier des conseils de femmes māori plus âgées qui lui ont transmis leur savoirfaire technique ainsi que les valeurs associées aux divers types de tissages. Mais après s'être blessée au dos, elle a dû faire face à des contraintes physiques qui l'ont poussée à apprendre la technique du tāniko en autodidacte, technique qu'elle a ensuite perfectionné avec l'aide de l'une des deux fondatrices du groupe de tissage qu'elle fréquentait. Le tāniko consiste à tisser manuellement, sans utiliser d'outils, en entrelaçant des fils colorés et d'autres neutres de manière à réaliser une trame permettant de fixer les plumes des manteaux korowai (des manteaux māori prestigieux, recouverts de plumes) ou de créer des motifs géométriques également appelés tāniko. Deborah Duncan utilise cette technique à la fois pour produire des motifs tāniko et des korowai (photo 33), (fig. 34). 


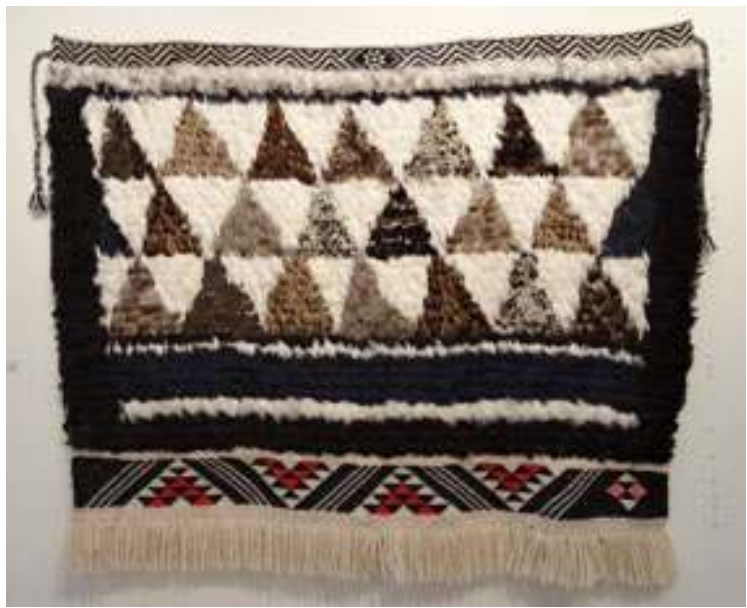

Deborah Duncan, 2013, laine, coton, plumes. Extrait de « Korowai Exhibition », Depot Artspace, Devonport, Auckland

(c) C. Pellini, 24 juin 2013

\section{Technique tāniko}

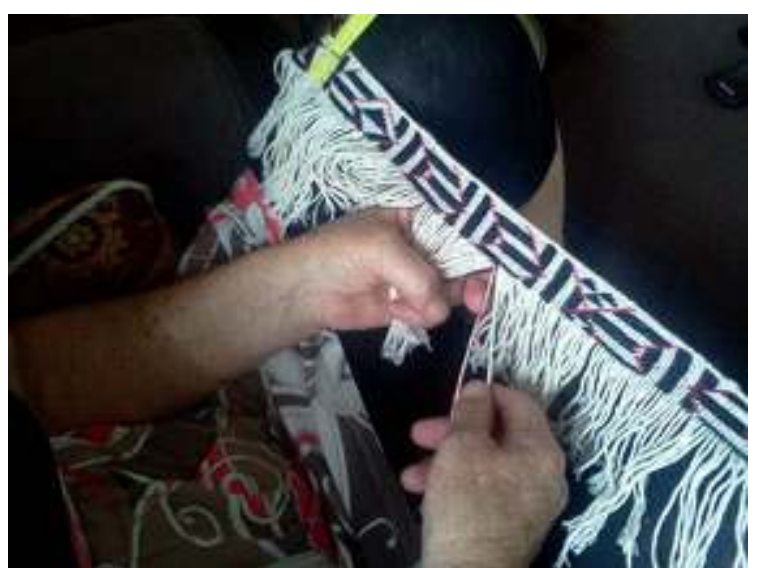

Tissage d'une ceinture en laine par Deborah Duncan

(c) C. Pellini, 17 novembre 2013, Puhinui, Papatoetoe, Auckland

L'élaboration de motifs tāniko demande un grand savoir-faire. En effet, il faut calculer précisément quels fils doivent passer à l'avant-plan et à quel rang et Deborah Duncan s'aide alors de schémas qu'elle a dessinés au préalable (fig. 35). 
Détail du schéma élaboré pour la réalisation du motif tāniko

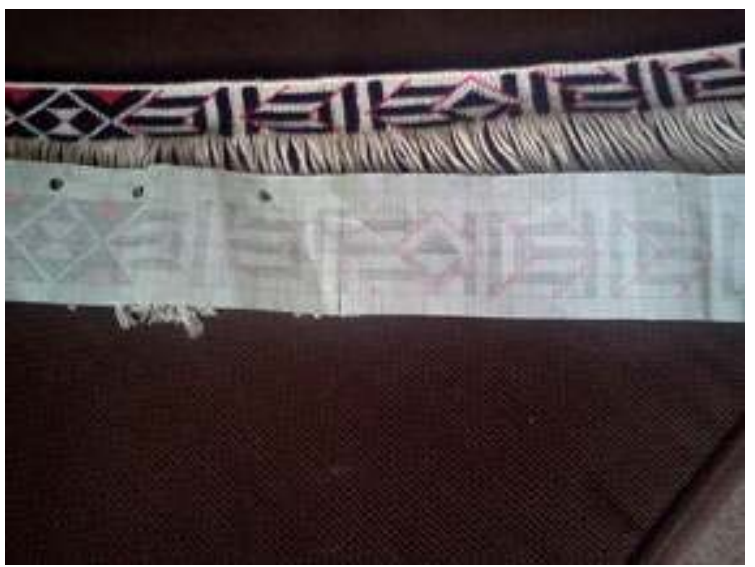

Puhinui, Papatoetoe, Auckland

(C) C. Pellini, 17 novembre 2013

26 Ses productions semblent très semblables à celles précoloniales, mais un examen plus attentif permet de déceler des innovations. Parmi les plus subtiles: citons le remplacement, par de la laine, des fibres végétales utilisées de façon traditionnelle. De manière plus évidente, Deborah Duncan fait parfois usage de perles en plastique (fig. 36) et a réalisé un kete en fil de fer barbelé alors que les kete sont des paniers traditionnellement produits en entrelaçant des fibres issues de plantes endémiques néo-zélandaises (fig. 37).

\section{Kowhaiwhai Bling}

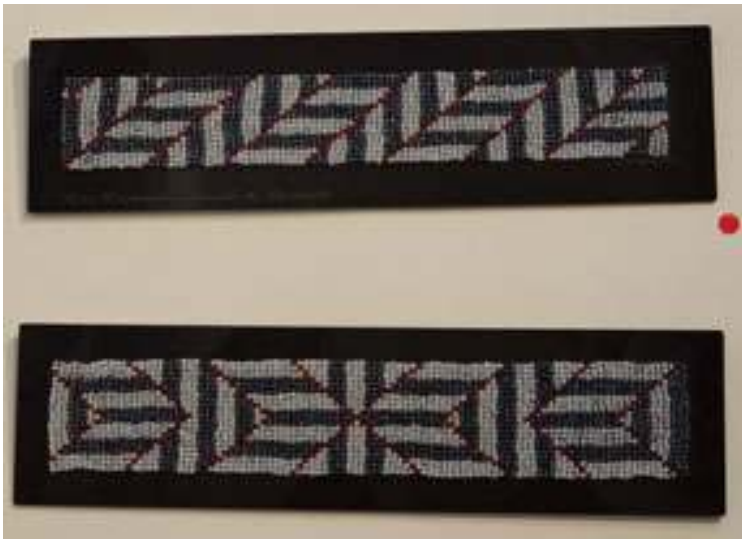

Deborah Duncan, 2013, perles et plexiglas. Extrait de « Korowai Exhibition », Depot Artspace, Devonport, Auckland

(c) C. Pellini, 24 juin 2013 


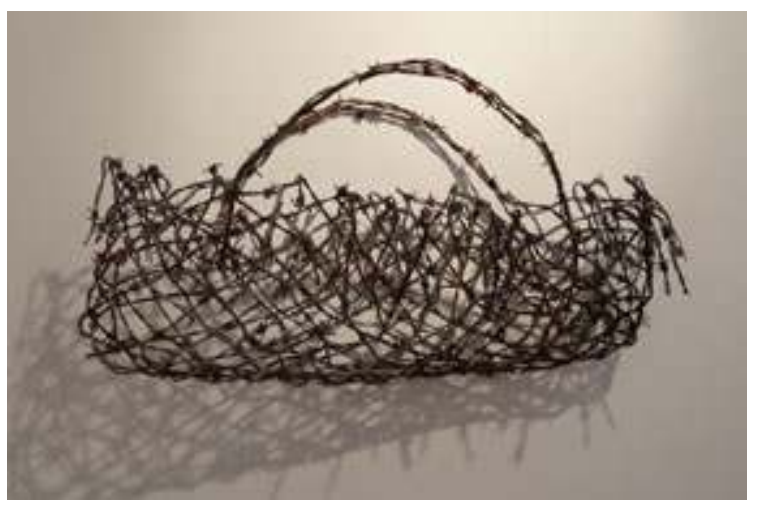

Deborah Duncan, 2013, fil barbelé. Extrait de « Korowai Exhibition», Depot Artspace, Devonport, Auckland

(C) C. Pellini, 24 juin 2013

Cette œuvre qui s'intitule Māori woman in a white skin fait simultanément référence à un récit mythique māori, au parcours de l'artiste et à une problématique identitaire. En effet, le kete renvoie au mythe des trois paniers de la connaissance. Ce récit raconte comment Tane, l'un des fils de Papatūānuku (la Terre-Mère) et Ranginui (le Ciel-Père) a transmis aux hommes trois paniers contenant les savoirs sacrés et ésotériques, ceux positifs pour l'humanité et ceux malfaisants (Best 1934 : 38). Pour Deborah Duncan, ce kete symbolise le savoir qu'on a tenté de lui cacher. Son père ayant souffert de discriminations, il ne voulait pas que cette situation se reproduise pour ses enfants et il a alors tenté de leur dissimuler leurs origines māori et de couper les liens avec leur famille māori. Deborah Duncan a souffert de cette situation. Ayant une peau très claire, elle a également subi le rejet de la part d'autres Māori qui la rattachaient au clan des « oppresseurs » occidentaux. Elle a alors dû lutter pour accéder au savoir la concernant et elle a créé ce kete en barbelés pour symboliser cette difficulté tout en le transformant en réceptacle destiné à accueillir les expériences négatives qu'elle a vécues et ainsi les laisser derrière elle (entretien avec Deborah Duncan le 19 août 2013). Il s'agit donc d'une œuvre qui fait référence à la culture māori mais de manière à refléter aussi les expériences vécues par l'artiste. Le tissage se prête bien à ce genre de création puisqu'il permet de faire aisément référence à la tradition artistique māori, et en particulier à la tradition artistique féminine. Alors, même si parmi les artistes rencontrées, Deborah Duncan était la seule à réaliser des korowai, des kete et des taniko, beaucoup d'autres font référence au tissage en créant des œuvres non textiles. Ainsi, pour ne citer qu'un seul exemple, dans son installation Whare Taonga (2013) réalisée en collaboration avec Rona Ngāhuia Osborne, Alexis Neal entrelace des bandelettes de papier imprimé en obtenant de la sorte des motifs tissés traditionnels. Elle crée ainsi des whariki (tapis de sol) qui diffèrent de leurs modèles classiques tant au niveau des matériaux que dans leur disposition. En effet, les whariki sont normalement composés de fibres végétales entrelacées et sont disposés au sol. Ils pouvaient notamment être placés sous le couchage de personnes de haut rang. Dans Whare Taonga ${ }^{16}$, les whariki d'Alexis Neal ne se trouvent pas sur le sol mais accrochés aux murs bien que leur partie inférieure touche le sol. Ils peuvent alors être vus comme des panneaux tukutuku, des panneaux tressés se trouvant sur les parois des maisons de réunion. L'artiste propose une double référence aux productions traditionnelles en alliant une technique moderne 
(l'impression de grandes feuilles de papier découpées en bandelettes) et un savoir-faire traditionnel (entretien avec Alexis Neal, le 26 juin 2013) (fig. 38), (fig. 39).

\section{Whare Taonga}

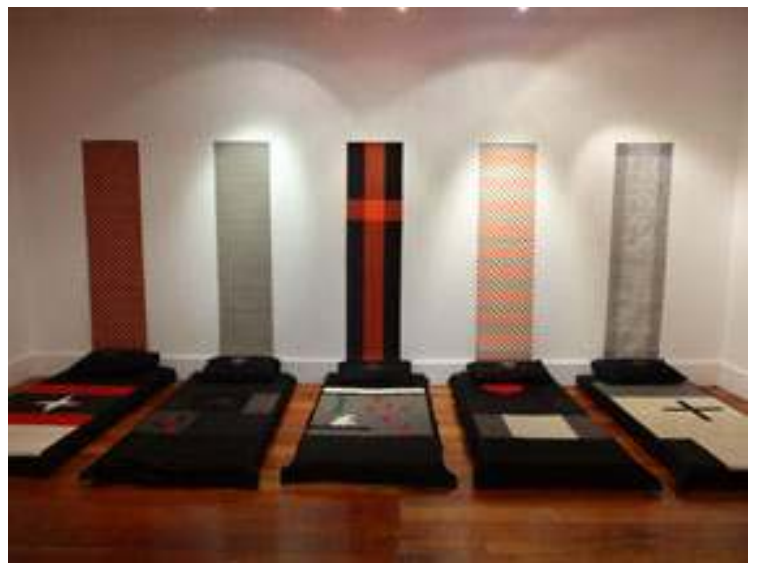

Alexis Neal et Rona Ngāhuia Osborne, 2013, Corban Estate Arts Centre, Henderson, Auckland (C) C. Pellini, 26 juin 2013

\section{Whariki (détail)}

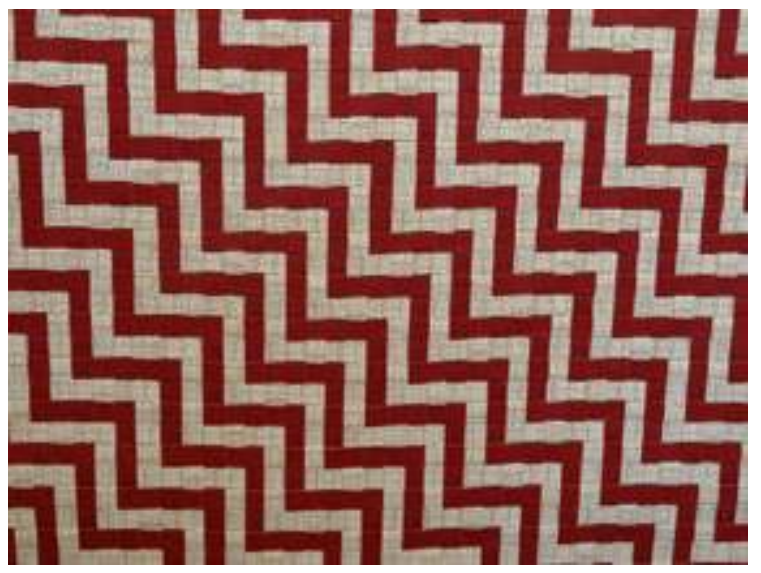

Alexis Neal, 2013. Extrait de l'installation «Whare taonga », d'Alexis Neal et Rona Ngāhuia Osborne, 2013, Corban Estate Arts Centre, Henderson, Auckland

(c) C. Pellini, 26 juin 201

Les réalisations présentées dans cet article témoignent de l'adoption de techniques et de matériaux très différents de ceux employés par les Māori avant la colonisation ainsi que de l'impact de la culture des colons. Mais l'adoption de pratiques et de matériaux introduits par la colonisation n'est pas considérée comme une renonciation à la culture māori. Au contraire, les artistes parlent de la faculté d'adaptation détenue par les Māori avant même le contact avec les Européens. Elles rejoignent ici les propos de certains chercheurs qui montrent comment à leur arrivée en Nouvelle-Zélande les Māori se sont adaptés à leur nouvel environnement. Belich (2001), Walker (2004) et King (2003), pour ne citer qu'eux, décrivent une société non uniforme formée de groupes disparates, reflets des nombreuses mutations qui se sont produites au cours de leur histoire. Avec la colonisation, des transformations inévitables et souvent brutales ont eu lieu mais la culture māori ne s'est pas éteinte pour autant, contrairement à ce que prévoyaient des 
anthropologues du début du $\mathrm{xx}^{\mathrm{e}}$ siècle qui considéraient les Māori comme une « dying race » (Zaitz 2009 : 91). Les réalisations contemporaines des artistes māori témoignent de la vitalité de cette culture : les œuvres sont novatrices en termes de sujets, de matériaux et de techniques tout en restant liées à la tradition culturelle māori par certains indices visuels et/ou par le sens que leurs auteurs y insufflent. Mais ce lien peut parfois être encore plus explicite lorsqu'il se manifeste par l'adoption de matériaux et de techniques précoloniales. L'exemple le plus flagrant est celui du tissage.

Les matériaux choisis sont souvent significatifs, ils sont en adéquation avec la démarche intellectuelle des artistes. Mais ces dernières ont également dû se plier à des contraintes matérielles et adapter quelquefois leurs pratiques aux matériaux dont elles pouvaient disposer. Ainsi, Vicky Thomas m'a expliqué qu'elle aimerait utiliser un appareil photographique plus coûteux mais elle n'a pas les moyens financiers pour l'acquérir. Hera Johns aime beaucoup l'argile car c'est non seulement un élément lié à Papatūānuku, mais c'est aussi une matière peu chère qu'elle peut réutiliser en cas de raté et qui ne présente pas de danger de toxicité pour ses enfants. Avant de les doter d'un caractère critique, Tracey Tawhiao a commencé à utiliser des journaux comme support de ses œuvres car il s'agissait d'un matériau abondant et peu onéreux. Il ne s'agit là que de quelques exemples mais toutes les artistes rencontrées ont dû faire face à ce genre de considérations et mettre au point des techniques leur permettant de concilier créativité et praticité. De même, des artistes comme Hera Johns ont développé des stratégies pour créer des œuvres novatrices sans briser certains tapu entourant les pratiques artistiques traditionnelles.

En ne se tournant pas vers l'art traditionnel pour exprimer leur attachement à la culture māori mais en produisant des œuvres qui mêlent des techniques, des motifs et des matériaux issus de l'art māori précolonial et des éléments observables dans des productions contemporaines occidentales, les femmes artistes māori sont à l'origine de créations singulières qui reflètent leurs identités à multiples facettes. Elles œuvrent pour faire entendre les voix des femmes māori vivant dans un milieu urbain pākehā, et perpétuent le rôle des artistes māori traditionnels qui, selon Jackson (1972: 35-36), tentaient de résoudre les tensions et les conflits qui agitaient la société en produisant de nouvelles formes et en agissant ainsi sur la structure de la vie sociale.

\section{BIBLIOGRAPHIE}

Balasinski, J. \& L. Mathieu dir. 2006 Art et Contestation. Rennes : Presses universitaires de Rennes. Barrow, T. 1968 [1964] The Decorative arts of the New Zealand Māori. Kyodo : A.H. \& A.W. Reed. Belich, J. 2001 [1996] Making people, a history of the New Zealanders. Honolulu : University of Hawai'i Press.

Best, E. 1934 The Māori as he was : a brief account of life as it was in Pre-european days. Wellington : Dominion Museum. 
- 1924 The Māori. New York : AMS Press.

Bilge, S. 2009 « Théorisations féministes de l'intersectionnalité », Diogène 225 : 70-88.

Bowden, R. 1979 « Tapu and Mana : Ritual authority and political power in traditional Māori society » The Journal of Pacific History 14(1) : 50-61.

Bradley, W. \& C. Esche dir. 2007 Art and social change, a critical reader. London : Tate Publishing.

Charles-Rault, J. 2005 « Une nouvelle génération d'artistes en Nouvelle-Zélande » in Groupe de Recherche Identités et Cultures, équipe Stratégie des Pacifiques dir. Art et Littérature dans le Pacifique, Tradition et Modernité, tome II. Paris : L'Harmattan : 91-106.

Crenshaw, K. 1989 « Demarginalizing the intersection of race and sex : a black feminist critique of antidiscrimination doctrine, feminist theory and antiracist politics ", University of Chicago Legal Forum : 139-167.

Curchin, K. 2011 « Pākehā women and Māori protocol : the politics of criticising other cultures », Australian Journal of Political Science 46(3) : 375-388.

Davis, K. 2008 «Intersectionality as buzzword, a sociology of science perspective on what makes a feminist theory successful », Feminist Theory 9(1) : 67-85.

Denis, A. 2008 « Intersectional analysis, a contribution of feminism to sociology », International Sociology Review of Books 23(5) : 677-694.

Diamond, J. 1999 « Hine-Titama : Māori contributions to feminist discourses and identity politics », Australian Journal of Social Issues 34(4) : 301-317.

Gagné, N. 2013 Being Māori in the city : indigenous everyday life in Auckland. Toronto : University of Toronto Press.

Gell, A. 2009 L'Art et ses agents - Une théorie anthropologique. Paris : Presses du Réel.

Glowczeswski, B. et R. Henry. 2007 Le Défi indigène. Entre spectacle et politique. Montreuil : Aux lieux d'être.

Graille, C. 2003 « Primitifs d'hier, artistes de demain : l'art kanak et océanien en quête d'une nouvelle légitimité », Ethnologies comparées 6.

Gwynne, C. R. 2009 « Artistic practices, representations of Māori women and the paradox of Kaupapa Māori », MAI Review, 1 Intern Research Report 6 : 1-11.

Hamilton-Pearce J. 2009 Mana Wāhine in information technology : Ngā Kaiwhatu Kākahu Me Te Kākahu. Thèse de doctorat, School of Computing and Mathematical Sciences, AUT University, Te Wānanga Aronui o Tāmaki Makaurau.

Hanson, A. 1982 « Female pollution in Polynesia? », The Journal of the Polynesian Society, 91(3): 335-382.

Heuer, B. 1972 Māori women. Wellington : AH \& AW Reed.

Hibbs, S. 2006 « The uniquely female art of karanga », Social Work Review 18(2) : 3-8.

Ihimaera, W., S. Adsett \& C. Whiting dir. 1996 Mataora, the living face. Contemporary māori art, Te Wake Toi. Auckland : David Bateman Ltd.

Jackson, M. 1972 « Aspects of symbolism and composition in Māori Art », Bijdragen tot de Taal-, Land- en Volkenkunde (Journal of the Humanities and Social Sciences of Southeast Asia), 128(1) : 33-80.

King, M. 2003 The Penguin history of New Zealand. Auckland : Penguin Books. 
McCarthy, C. 2007 Exhibiting Maori, a history of colonial cultures of display. New York : Berg Publishers.

Mead, S. H. M. 1975 « The origins of Māori art : Polynesian or Chinese ? », Oceania 45(3) : 173-211. - 1997 « The Nature of taonga » in Mead, S. H. M. dir. Landmarks, Bridges and Visions : Aspects of Maori culture. Wellington : Victoria University Press.

- 2003 Tikanga Māori : Living by Māori values. Wellington : Huia Publishers.

Melbourne, T. W. 2011, Te wairua kōmingomingo o te Māori, The spiritual whirlwind of the Māori. Thèse de doctorat, Palmerston North : Massey University.

Metge, J. 2002 « Returning the gift - utu in intergroup relations : in memory of Sir Raymond Firth », Journal of the Polynesian Society, 111(4) : 311-338.

- 2004 [1967] Rautahi : The Māoris of New Zealand. Londres : Routledge.

Nash, J. C. 2008 « Re-thinking intersectionality », Feminist review 89 : 1-15.

Negash, G. « Art invoked : a mode of understanding and shaping the political », International Political Science Review 25(2) : 185-201.

Neich, R., 2004 [1994] Painted histories : early Māori figurative painting. Auckland : Auckland University Press.

Orange, C. 2011 [1987] The Treaty of Waitangi. Wellington : Bridget William Books.

Panoho, R. 2004 « Smoke and mirrors. Subterfuge and reflection in Māori art », Third Text 18(3): 283-291.

Patterson, J. 1992 Exploring Māori values. Palmerston North : The Dunmore Press.

Pihama, L. 2001 Tihei Mauri Ora: Honouring our voices. Mana Wahine as a Kaupapa Māori theoretical framework. Thèse de doctorat, Auckland : University of Auckland.

Schwimmer, E. 1972 [1968] « The aspirations of the contemporary māori », in E. Schwimmer dir. The Māori people in the nineteen-sixties, a symposium. Auckland : Longman Paul Limited : 9-64.

Simmonds, N. 2011 « Mana Wahine : decolonising politics », Women's Studies Journal 25(2) : 11-25.

Sissons, J. 2010 « Building a house society : the reorganization of Māori communities around meeting houses », Journal of the Royal Anthropological 16 : 372-386.

Smith, J. 2001 « Multiculturalism and biculturalism art education in New Zealand », Ace Paper, 8 : 84-102.

Smith, L. T. 1999 Decolonizing methodologies : research and indigenous people. London : Zed Books. Smith, H. 2002 Taiawhio : Conversations with contemporary Māori artists. Wellington : Te Papa Press. Statistics New Zealand 2013 « 2013 census quick stats about Māori », Statistics New Zealand. [En ligne], mis en ligne le 3 décembre 2013. URL : http://www.stats.govt.nz/Census/2013-census/ profile-and-summary-reports/quickstats-about-māori-english.aspx. Consulté le 10 juin 2014.

Stevenson, K. 2002 « The island in the urban : contemporary Pacific art in New Zealand » in Anita H. et al dir. Pacific Art, Persistence, Change and Meaning. Honolulu : University of Hawai'i Press : 404-414.

Tamarapa, A. \& P. Wallace 2013 « Māori clothing and adornment - kākahu Māori - Weaving traditions and technique », Te Ara - the Encyclopedia of New Zealand. [En ligne], mis à jour le 
22 août 2013. URL : http://www.TeAra.govt.nz/en/māori-clothing-and-adornment-kakahumāori/page-3. Consulté le 20 août 2014.

Thomas, N. 1995 L'Art de l'Océanie. Paris : Thames \& Hudson.

- 1999 Possessions. Indigenous art/colonial culture. London : Thames \& Hudson.

Walker, R. 2004 Ka Whawhai Tonu Matou : Struggle Without End. Auckland : Penguin Books, 2de édition.

Zaitz, C. L. 2009 Matters of life and death : a comparative analysis of content in Māori traditional and contemporary art and dance as a reflection of fundamental Māori cultural issues and the formation and perpetuation of Māori and non-Māori cultural identity in New Zealand. Thèse de doctorat, Florida : Florida Atlantic University.

\section{NOTES}

1. Au sujet de ce traité et des polémiques liées aux divergences existant entre la version māori et celle anglaise en ce qui concerne les notions de souveraineté et de propriété des terres, voir Orange 2011 [1987].

2. Le terme « pākehā» fait référence aux néo-zélandais non-māori d'origine européenne.

3. Dans cet article, l'expression « art traditionnel » désigne essentiellement des pratiques ou des artefacts produits avant ou lors de la colonisation et ayant été retrouvés et datés par les archéologues, décrits par les anthropologues, les missionnaires, les commerçants, les voyageurs occidentaux de l'époque ou étant mentionnés dans les traditions orales māori. Mais il serait erroné de considérer qu'il existe réellement une entité figée nommée " art traditionnel » : même avant la colonisation, l'art māori était en constante évolution, différents styles se sont succédés ou ont vu le jour au sein de groupes divers (voir Jackson 1972; Mead 1975 ; Neich 2004 [1994] : 9-10). Pour corser encore la définition, l'expression « art traditionnel » peut aussi qualifier des œuvres faites de nos jours. Dans ce cas il s'agit de réalisations produites en utilisant des techniques, des matériaux et des motifs pensés par les Māori comme étant hérités des ancêtres. Certains anthropologues ont remis en question la pertinence des catégories « art traditionnel » et «art contemporain» (voir en particulier Stevenson 2002: 406 et Graille 2003: 6). Mais pour faciliter la compréhension de mon propos, j'emploie tout de même dans ce texte l'expression «art traditionnel » pour évoquer les œuvres et les pratiques artistiques du passé ou celles contemporaines entretenant une forte ressemblance avec les productions précoloniales.

4. Ceci fait écho à certaines de leurs affirmations au cours de nos entretiens : bien que toutes les artistes aient également des origines européennes, elles se considèrent le plus souvent comme Māori. Même si elles s'intéressent à leurs autres origines, elles mettent l'accent sur leur côté māori car il s'agit du peuple autochtone de la Nouvelle-Zélande et il leur semble important de revendiquer cet héritage dont leurs parents ont fréquemment été dépossédés ou honteux. En effet, le contexte actuel leur offre la possibilité de s'affirmer plus facilement comme Māori alors que ce n'était pas le cas pour la génération de leurs parents qui ont connu une politique d'assimilation et pour lesquels le fait de se revendiquer Māori engendrait souvent des discriminations.

5. Dans son ouvrage L'Art et ses agents - Une théorie anthropologique, Alfred Gell nous invite à considérer les œuvres comme les produits de différentes intentionnalités - celles de l'artiste, du sujet représenté, du commanditaire et du public - possiblement contradictoires.

6. Sur cette théorie, voir notamment les textes de Crenshaw 1989, Davis 2008, Denis 2008, Nash 2008 et Bilge 2009. 
7. Certains anthropologues considéraient qu'à cause de leur caractère noa les femmes étaient perçues dans la société māori comme dangereuses et potentiellement malfaisantes puisqu'elles pouvaient corrompre le tapu entourant certains êtres, éléments et activités (Best 1924 ; Heuer 1972 ; Bowden 1979; Hanson 1982). Des chercheuses māori se sont élevées contre cette conception en parlant de la complémentarité des valeurs tapu et noa que l'on peut grossièrement traduire respectivement par «sacré » et «profane ». Elles expliquent que le terme noa est en réalité associé à la capacité d'ôter des restrictions contraignantes et désigne un état sécuritaire. L'état noa n'est pas plus péjoratif que celui tapu et il est nécessaire d'obtenir un équilibre entre les deux (Metge 2004 [1967] ; Pihama 2001 ; Mead 2003).

8. Selon de nombreux récits māori, Papatūānuku, la Terre Mère, et Ranginui, le Ciel Père, sont considérés comme étant à l'origine de toutes les divinités dont celle ayant créé les humains. Un lien généalogique relie donc tous les Māori à Papatūānuku (Patterson 1992 : 98) et les différentes iwi (tribus), hapu (sous-tribu) et whānau (familles étendues) sont liés à des territoires particuliers sur lesquels leurs ancêtres se sont établis (Patterson 1992 : 88). Les divers groupes se considèrent comme les tangata whenua d'un territoire, littéralement "people who belong to the land» (Sissons $2010: 384$ ).

9. Nicholas Thomas décrit ces motifs curvilinéaires en expliquant qu'ils «combinent des enchevêtrements de spirales entières ou brisées à d'autres éléments, séparés ou associés à des koru ", les koru apparaissant " habituellement sous forme d'arborescences peintes en blanc. Ils naissent à l'extrémité de figures dissymétriques évoquant les quartiers de lune; les espaces intermédiaires sont alternativement remplis en rouge et en noir » (Thomas 1995 : 71-72).

10. La spirale est un élément clé dans la culture māori. Elle évoque l'idée d'un mouvement perpétuel entre passé et présent et symbolise une idée d'harmonie et de croissance basées sur des fondations solides ancrées dans le passé (Gagné 2013 : 339).

11. Au sujet de la création de nouveaux réseaux urbain suite au phénomène de migration des Māori dans les villes qui a engendré l'éclatement des groupes familiaux traditionnels, voir le livre de Natacha Gagné Being Māori in the City : Indigenous Everyday Life in Auckland (2013).

12. Taonga est un terme māori généralement traduit par «trésor culturel ». Il peut désigner des biens précieux matériels transmis de génération en génération et acquérant ainsi au fil du temps davantage de mana (prestige), mais également des ressources comme la terre ou des éléments immatériels tels que des chants et des danses (Metge 2002 : 335). Pour en savoir plus sur les taonga, voir Mead 1997.

13. Robyn Kahukiwa est l'une des premières femmes artistes māori à avoir acquis une grande renommée. C'est une artiste et une illustratrice qui est à l'origine, entre autres, de peintures et de dessins abordant des thèmes tels que la décolonisation et les récits cosmologiques, mythiques et historiques māori, mettant en avant des figures féminines māori. À son sujet, voir notamment Diamond, 1999.

14. George Nuku est un artiste māori qui pratique la sculpture et la gravure. Il crée des motifs rappelant ceux de la sculpture et du tissage māori traditionnel en travaillant avec des matériaux très divers : jade, os, bois, coquillages, pierre mais aussi polystyrène et plexiglas.

15. Cette notion de confort et d'inconfort est très souvent revenue au cours des discussions avec diverses artistes, c'est un point également relevé par des anthropologues comme Natacha Gagné (2013: 73-75).

16. Cette installation recrée en le revisitant l'espace intérieur d'une wharenui (maison de réunion). Les whariki d'Alexis Neal sont accrochés à la verticale sur les murs de la galerie d'exposition. Ils se trouvent au-dessus de matelas placés sur le sol. Ces couchages sont recouverts de couvertures et de coussins ornés de décorations textiles symboliques réalisées par Rona Ngāhuia Osborne, faisant notamment référence au contexte historique et colonial néo-zélandais et à la perte des terres māori. 


\section{RÉSUMÉS}

Peu d'anthropologues se sont intéressés à l'art contemporain des Māori de Nouvelle-Zélande, et encore moins aux productions artistiques féminines māori contemporaines. Ces dernières, ainsi que les pratiques des artistes et les discours qui les accompagnent, méritent pourtant d'être étudiés d'un point de vue ethnologique. Dans ce numéro dédié à la bricologie, les œuvres contemporaines des artistes māori sont examinées en attachant une attention particulière aux techniques et aux matériaux. Ceux-ci révèlent la multiplicité des sources auxquelles puisent les artistes pour engendrer des créations aux influences multiples mais pourtant présentées sous l'étiquette d'«art māori». Les productions et les discours des femmes māori témoignent également de la particularité de leur statut et de leur positionnement identitaire qui peut être être appréhendée par la théorie de l'intersectionnalité : les discriminations liées au genre s'ajoutent aux discriminations raciales, entre autres, et sont alors à l'origine d'expériences singulières différentes de celles vécues par les artistes masculins māori. Les œuvres des femmes artistes māori présentent ainsi un «bricolage » à double niveau : outre celui également visible chez leurs homologues masculins qui créent des œuvres singulières en utilisant et reconfigurant des éléments provenant de mondes sociaux différents, un deuxième « bricolage » est discernable cette fois au niveau des identités de genre. Les productions et les discours portant sur les parcours de vie des artistes permettent d'appréhender les identités multiples de ces femmes qu'elles concilient en mettant en place diverses stratégies. Afin d'examiner ce double «bricolage ", les productions et les parcours de vie détaillés de quatre artistes - Tracey Tawhiao, Hera Johns, Vicky Thomas et Deborah Duncan - sont présentés dans cet article.

Few anthropologists have been interested in the contemporary art of the Māori of New Zealand, and even less in the works produced by Māori women artists. However, these artworks, as well as the practices of the artists and accompanying discourses, deserve to be considered from an ethnological point of view. In this issue, contemporary Māori women's creations are examined with particular attention to techniques and materials, which reveal the multiplicity of the sources from which the artists draw to generate artworks with various influences, and yet presented under the "Māori art" label. Māori women's artistic productions and discourses also reflect the peculiarity of their status and identity positioning that can be understood through the theory of intersectionality: gender discriminations interact with racial discriminations, among other factors, to produce singular experiences different from Māori male artists' experiences. In this paper, the works and life trajectories of four Māori women artists - Tracey Tawhiao, Hera Johns, Vicky Thomas and Deborah Duncan - will be presented by focusing on a double "bricolage": one regarding the reconfiguration of materials, practices and patterns coming from different social worlds to create original artworks, and one related to gender identities.

\section{INDEX}

Mots-clés : Art contemporain māori, femmes māori, femmes artistes, biculturalisme, techniques et matériaux, art et revendications, problématiques identitaires, intersectionnalité

Keywords : Contemporary Māori art, Māori women, women artists, biculturalism, techniques and materials, protest art, identity issues, intersectionality 
AUTEUR

CATHERINE PELLINI

Université d'Ottawa et Aix Marseille Université CNRS, EHESS, CREDO UMR 7308 